\title{
Detailed characteristics of drop-laden mixing layers: Large eddy simulation predictions compared to direct numerical simulation
}

\author{
Nora Okong'o, ${ }^{1}$ Anthony Leboissetier, ${ }^{1}$ and Josette Bellan ${ }^{1,2, a)}$ \\ ${ }^{1}$ Jet Propulsion Laboratory, California Institute of Technology, Pasadena, California 91109-8099, USA \\ ${ }^{2}$ California Institute of Technology, Pasadena, California 91125, USA
}

(Received 20 February 2008; accepted 21 July 2008; published online 28 October 2008)

\begin{abstract}
Results are compared from direct numerical simulation (DNS) and large eddy simulation (LES) of a temporal mixing layer laden with evaporating drops to assess the ability of LES to reproduce detailed characteristics of DNS. The LES used computational drops, each of which represented eight physical drops, and a reduced flow field resolution using a grid spacing four times larger than that of the DNS. The LES also used models for the filtered source terms, which express the coupling of the drops with the flow, and for the unresolved subgrid-scale (SGS) fluxes of species mass, momentum, and enthalpy. The LESs were conducted using one of three different SGS-flux models: dynamic-coefficient gradient (GRD), dynamic-coefficient Smagorinsky (SMD), and constantcoefficient scale similarity (SSC). The comparison of the LES with the filtered-and-coarsened (FC) DNS considered detailed aspects of the flow that are of interest in ignition or full combustion. All LESs captured the largest-scale vortex, the global amount of vapor emanating from the drops, and the overall size distribution of the drops. All LESs tended to underpredict the global amount of irreversible entropy production (dissipation). The SMD model was found unable to capture either the global or local vorticity variation and had minimal small-scale activity in dynamic and thermodynamic variables compared to the FC-DNS. The SMD model was also deficient in predicting the spatial distribution of drops and of the dissipation. In contrast, the GRD and SSC models did mimic the small-scale activity of the FC-DNS and the spatial distribution of drops and of the dissipation. Therefore, the GRD and SSC models are recommended, while the SMD model seems inappropriate for combustion or other problems where the local activity must be predicted. (C) 2008 American Institute of Physics. [DOI: 10.1063/1.2990758]
\end{abstract}

\section{INTRODUCTION}

The modeling of spray combustion remains an important research topic as there is no consensus on what is the optimal way to achieve this goal. Currently, there are three methodologies for spray modeling: direct numerical simulation (DNS), large eddy simulation (LES), and Reynolds average Navier-Stokes (RANS). Of these three methodologies, RANS is the oldest, best documented and its limitations are well known: it cannot resolve the small flow structures that are crucial in reaction-rate prediction. For this reason, the present study will only address DNS and LES.

DNS is a methodology wherein all scales of the flow are resolved, but due to the number of scales which increase with the Reynolds number value, Re, and due to computer central processing unit (CPU) and memory limitations, it is currently not possible to exercise it for Re values that are of typical interest in practical applications. Originally developed for atmospheric single-phase (SP) incompressible flows, DNS has been extended to much more computationally complex situations, including two-phase (TP) flows. For TP flows with particles that are much smaller than the Kolmogorov scale and which have a volumetrically small loading $\left(\simeq 10^{-3}\right)$, Boivin et al. ${ }^{1}$ showed that the drops can be

\footnotetext{
${ }^{a)}$ Telephone: 1-818-354-6959. Fax: 1-818-393-6682. Electronic mail: josette.bellan@jpl.nasa.gov.
}

treated as point sources of mass, momentum and energy from the gas-phase perspective. In such situations, it is appropriate to perform simulations using a gas-phase resolution that is adequate for SP flow using an Eulerian framework to describe the gas phase and a Lagrangian framework to track the drops. The terminology DNS, while not strictly accurate, is traditionally applied to such simulations. Several recent studies have used this DNS methodology, ${ }^{1-5}$ and it has more recently been used for DNS of a transitional temporal mixing layer with evaporating drops. ${ }^{6-10}$

LES, in which only the large scales must be resolved, is a promising methodology for situations not accessible in DNS because LES does not have Re value limitations in turbulent free shear flows. As such, LES has been recently used to study various aspects of evaporating and/or chemically reacting sprays; ${ }^{11-14}$ in all LES involving combustion (e.g., Ref. 15), prediction of mixing is a prerequisite for that of accurate heat release. The LES gas-phase equations are derived by filtering the DNS gas-phase equations, leading to unclosed terms: the subgrid-scale (SGS) fluxes that arise from the convective terms and the filtered source terms (FSTs) that embody the effect of the drops on the resolved flow field. For volumetrically dilute TP flows, LES may be conducted not only with reduced flow field resolution compared to DNS but also with "computational" drops to represent the physical drops. This reduction in the physical drop field to a representative drop field is in concert with the re- 
duction in flow scales through modeling. ${ }^{8}$ Several LESs of TP flows have considered an incompressible gas phase laden with small solid particles, with one-way ${ }^{16-21}$ or two-way ${ }^{11,12,16,21}$ coupling, and used physical or computational particles whose evolution was entirely governed by the resolved flow field, that is, neglecting SGS effects on drop evolution. ${ }^{22}$ Of note is that for TP flows with evaporating liquid drops, the liquid is $O\left(10^{3}\right)$ times denser than the carrier gas, so the mass loadings can be significant $\left(\gtrsim 10^{-1}\right)$ despite a low volumetric loading; therefore, the drops may considerably influence the flow and this two-way coupling must be modeled. Furthermore, a compressible formulation is required due to the density changes induced by the vapor released through drop evaporation, and the thermodynamic variables are coupled through the equation of state, as well as through the heat and mass fluxes appearing in the energy and species equations. Therefore, appropriate modeling of the momentum, energy, and species SGS fluxes must be carefully assessed, with due consideration to the form of the energy equation. ${ }^{23-25}$ Using DNS of a temporal mixing layer ${ }^{8}$ and extending previous SGS TP flow models, ${ }^{7,26}$ LES models have been developed and tested, both a priori (using a DNS database $^{8}$ ) and a posteriori [by performing LES (Ref. 22)].

The interest here is to evaluate the LES ability to model aspects pertinent to predictions of mixing that will set the conditions for ignition in reactive flows; particularly, the study is devoted to analyzing the detailed characteristics of a mixing layer ${ }^{8,22}$ in terms of both global measures and spatial distributions at the DNS transitional time. The template for assessing the LES ability will be DNS. However, an unprocessed DNS database is not the proper template for comparison with LES. The proper template is the filtered-andcoarsened (FC) DNS: filtered to remove the small scales that are not computed in LES, and coarsened to reduce the number of nodes from DNS to those of LES. Thus, the FC-DNS field can be considered as a sample of the DNS field. This paper is organized as follows: In Sec. II, the highlights of the DNS formulation are presented, followed by a summary of the LES method in Sec. III. Section IV contains the analysis of the detailed characteristics predicted by LES, with conclusions in Sec. V.

\section{DNS METHODOLOGY}

Details of the DNS method have been given by Okong'o and Bellan, ${ }^{8}$ based on the formulation of Miller and Bellan. ${ }^{6}$ A brief summary is here presented of this formulation for the gas-phase and liquid-phase equations and of the flow configuration and the numerical procedure.

\section{A. Gas-phase equations}

The vector of gas-phase conservative variables $\phi$ $=\left\{\rho, \rho u_{i}, \rho e_{t}, \rho Y_{V}\right\}$ is defined and the flow field is denoted as $\phi$, where $\rho$ is the density, $u_{i}$ is the velocity in the $x_{i}$ coordinate direction, $e_{t}$ is the total energy, and $Y_{V}$ is the vapor (subscript $V$ ) mass fraction (the carrier gas, subscript $C$, mass fraction is $\left.Y_{C} ; Y_{C}+Y_{V}=1\right)$. The DNS gas-phase conservation equations are

$$
\frac{\partial \phi}{\partial t}+\frac{\partial\left(\phi u_{j}\right)}{\partial x_{j}}=\frac{\partial\left[\Theta_{j}(\phi)\right]}{\partial x_{j}}+S,
$$

where $S=\left\{S_{\mathrm{I}}, S_{\mathrm{II}, i}, S_{\mathrm{III}}, S_{\mathrm{I}}\right\}$ are source terms due to the action of the drops and

$$
\Theta_{j}(\phi)=\left\{0, \sigma_{i j}-p \delta_{i j}, \sigma_{i j} u_{i}-q_{j}-p u_{j},-j_{V j}\right\} .
$$

The thermodynamic variables to be computed from $\phi$ are the internal energy $\left(e=e_{t}-u_{i} u_{i} / 2\right)$, the pressure $(p)$, the temperature $(T)$, and the enthalpy $(h=e+p / \rho)$. The gas phase behaves according to a perfect gas, for which

$$
p(\phi)=\rho R(\phi) T(\phi),
$$

where $R(\phi)=Y_{V} R_{V}+Y_{C} R_{C}, R_{V}=R_{u} / m_{V}, R_{C}=R_{u} / m_{C}, R_{u}$ is the universal gas constant and $m_{C}$ and $m_{V}$ are the molar masses of the carrier gas and vapor, respectively. For the small temperature and pressure range to be simulated, the species heat capacities at constant pressure, $C_{p, C}$ and $C_{p, V}$, are assumed constant; then

$$
\begin{aligned}
& h(\phi)=h_{V} Y_{V}+h_{C} Y_{C}=C_{p}(\phi) T(\phi)+h_{V}^{0} Y_{V}, \\
& e(\phi)=e_{V} Y_{V}+e_{C} Y_{C}=C_{v}(\phi) T(\phi)+h_{V}^{0} Y_{V},
\end{aligned}
$$

where $C_{p}(\phi)=C_{p, V} Y_{V}+C_{p, C} Y_{C}, C_{v}=C_{p}-R$, and $h_{V}^{0}$ is the reference vapor enthalpy which accounts for the enthalpy difference between the vapor and carrier gas at the reference conditions $\left(T^{0}, p^{0}\right)$.

For Eq. (2), the viscous stress $\sigma_{i j}$, the vapor mass flux $j_{V j}$, and the heat flux $q_{j}$, are given by

$$
\begin{aligned}
& \sigma_{i j}(\phi)=2 \mu\left(S_{i j}-\frac{1}{3} S_{k k} \delta_{i j}\right), \\
& S_{i j}(\phi)=\frac{1}{2}\left(\frac{\partial u_{i}}{\partial x_{j}}+\frac{\partial u_{j}}{\partial x_{i}}\right), \\
& j_{V j}(\phi)=-\rho D \frac{\partial Y_{V}}{\partial x_{j}}, \\
& -\rho D\left(\frac{Y_{V}}{m_{V}}+\frac{Y_{C}}{m_{C}}\right)\left(m_{C}-m_{V}\right) \frac{Y_{V} Y_{C}}{p(\phi)} \frac{\partial p(\phi)}{\partial x_{j}}, \\
& q_{j}(\phi)=-\lambda \frac{\partial T(\phi)}{\partial x_{j}}+\left[h_{V}(\phi)-h_{C}(\phi)\right] j_{V j}(\phi),
\end{aligned}
$$

where $S_{i j}$ is the strain rate. The viscosity $\mu$, the diffusion coefficient $D$, and the thermal conductivity $\lambda$ are assumed constant; for a specified value of $\mu$, the $D$ and $\lambda$ values are inferred through the specified values of the Prandtl and Schmidt numbers, $\operatorname{Pr}=\mu C_{p} / \lambda$ and $\mathrm{Sc}=\mu / \rho D$.

\section{B. Drop (liquid-phase) equations}

The drop field is defined by $Z=\left\{X_{i}, v_{i}, T_{d}, m_{d}\right\}$ with position $X_{i}$, velocity $v_{i}$, temperature $T_{d}$, and mass $m_{d}$. Under the assumptions stated above, the evolution equations for the drops, in a Lagrangian frame, are ${ }^{6}$

$$
d Z / d t=\sum\left(\psi_{f}, \psi_{s}, Z\right)
$$




$$
\Sigma=\left\{v_{i}, \frac{F_{i}}{m_{d}}, \frac{Q+\dot{m}_{d} L_{V}(Z)}{m_{d} C_{L}}, \dot{m}_{d}\right\},
$$

where $F_{i}$ is the drag force, $Q$ is the heat flux, $\dot{m}_{d}$ is the evaporation rate, and $C_{L}$ is the heat capacity of the drop liquid. $L_{V}$ is the latent heat of vaporization, which, for calorically perfect gases, is a linear function of temperature, $L_{V}$ $=h_{V}^{0}-\left(C_{L}-C_{p, V}\right) T_{d}$. The drop evolution depends on the gasphase primitive variables, $\psi(\phi)=\left\{u_{i}, T, Y_{V}, p\right\}$, evaluated either at the drop surface (subscript $s$ ) or at the drop far field (subscript $f$ ). The far-field variables are taken as the gasphase primitive variables interpolated to the drop locations. The detailed expressions ${ }^{6}$ for $F_{i}, Q$, and $\dot{m}_{d}$ involve validated correlations for point drops that are based on the Stokes drag, with the particle time constant defined as ${ }^{27} \tau_{d}=\rho_{L} d^{2} / 18 \mu$, where $\rho_{L}$ is the density of the liquid and $d$ is the drop diameter (spherical drops; $m_{d}=\rho_{L} \pi d^{3} / 6$ ):

$$
\begin{aligned}
& F_{i}\left(\psi_{f}, Z\right)=\frac{m_{d}}{\tau_{d}} f_{1}\left(u_{i, f}-v_{i}\right), \\
& Q\left(\psi_{f}, Z\right)=\frac{m_{d}}{\tau_{d}} \frac{\mathrm{Nu}}{3 \operatorname{Pr}} C_{p, f} f_{2}\left(T_{f}-T_{d}\right), \\
& \dot{m}_{d}\left(\psi_{f}, \psi_{s}, Z\right)=-\frac{m_{d}}{\tau_{d}} \frac{\mathrm{Sh}}{3 \mathrm{Sc}} \ln \left(1+B_{M}\right) .
\end{aligned}
$$

Here, $f_{1}$ is an empirical correlation to correct the Stokes drag for finite drop Reynolds numbers and the mass transfer number is $B_{M}=\left(Y_{V, s}-Y_{V, f}\right) /\left(1-Y_{V, s}\right)$. The Nusselt $(\mathrm{Nu})$ and Sherwood (Sh) numbers are empirically modified for convective corrections to heat and mass transfer based on the RanzMarshall correlations. Except for $\tau_{d}$, which depends on $\mu$, Eqs. (12)-(14) depend essentially on ratios of transport properties through nondimensional numbers. Therefore, for the value of $\tau_{d}$ and thus for a given liquid and drop size, the value of $\mu$ determines the interaction time between drops and gas.

\section{Source terms}

Each drop acts as a point source ${ }^{6}$ of mass, momentum, and energy for the gas phase, with the drop source vector $S_{d}\left(\psi_{f}, \psi_{s}, Z\right)=\left\{S_{\mathrm{I}, d}, S_{\mathrm{II}, i, d}, S_{\mathrm{III}, d}, S_{I, d}\right\}$,

$$
\begin{aligned}
& S_{\mathrm{I}, d}=-\dot{m}_{d}, \quad S_{\mathrm{II}, i, d}=-\left[F_{i}+\dot{m}_{d} v_{i}\right], \\
& S_{\mathrm{III}, d}=-\left[F_{i} v_{i}+Q+\dot{m}_{d}\left(v_{i} v_{i} / 2+h_{V, s}\right)\right] .
\end{aligned}
$$

The drop sources in the Lagrangian frame are reconstructed in the Eulerian frame to obtain the gas-phase source vector $S(\psi, Z)$ for Eq. (1) using

$$
S(\psi, Z)=\sum_{\alpha=1}^{N_{\alpha}}\left(w_{\alpha} / V\right)\left[S_{d}\left(\psi_{f}, \psi_{s}, Z\right)\right]_{\alpha},
$$

where the summation is over the $N_{\alpha}$ drops within the local numerical discretization volume $V$, and the geometrical weighting factor $w_{\alpha}$ distributes the individual drop contributions to the corners of $V$ proportionally to the drop distance from those nodes. ${ }^{6}$ These source terms are then minimally

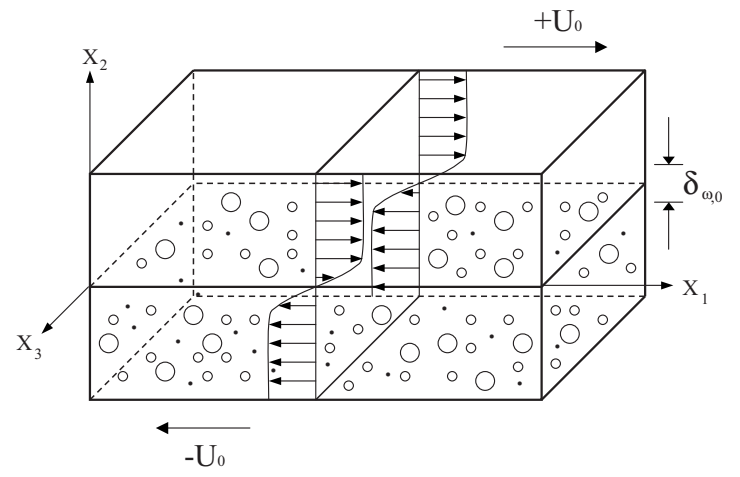

FIG. 1. Mixing layer configuration.

'smoothed' using a conservative operator so as to retain numerical stability of the Eulerian gas-phase fields; ${ }^{6}$ this smoothing is not a filter in that it does not remove flow scales but is required for successful simulations due to the 'spottiness' of the source terms.

\section{Flow configuration and numerical procedure}

The mixing layer geometric configuration is illustrated in Fig. 1, where the streamwise $\left(x_{1}\right)$, the cross-stream $\left(x_{2}\right)$, and the spanwise $\left(x_{3}\right)$ coordinates are shown. Periodic boundary conditions are used in the $x_{1}$ and $x_{3}$ directions, and adiabatic slip wall conditions ${ }^{28}$ are employed for the $x_{2}$ boundaries. Drops reaching the slip walls are assumed to stick to the wall but are otherwise transported according to Eq. (10). Initially, the gas phase consists only of the carrier gas (no vapor); the initial mean streamwise velocity has an error-function profile. To promote layer growth, the layer is initially perturbed so as to induce roll-up and pairing. The perturbations specify spanwise and streamwise vorticity fluctuations $^{6,29}$ and the evolution of the layer comprises two pairings for the four initial spanwise vortices to form a single vortex. The drops are initially distributed randomly throughout the $x_{2}<0$ domain; the initial velocity of each drop is the same as that of the gas phase at its location.

Several DNS computations were performed by Okong'o and Bellan ${ }^{8}$ out of which the selected cases for the present analysis are those with initial Reynolds number, $\operatorname{Re}_{0}$ $=\rho_{0} \Delta U_{0} \delta_{\omega, 0} / \mu$, of 600 (the specified Re value determines the $\mu$ value) and with initial mass loading, $M L_{0}$, of 0.2 (case TP600a2) and 0.5 (case TP600a5), listed in Table I. The initial number of drops, $N_{d, 0}$, is determined by $M L_{0}$. The initial vorticity thickness is $\delta_{\omega, 0}=\delta_{\omega}(0)$ where $\delta_{\omega}(t)$ $=\Delta U_{0} /\left(\partial\left\langle u_{1}\right\rangle / \partial x_{2}\right)_{\max }$; the brackets \langle\rangle denote averaging over homogeneous $\left(x_{1}, x_{3}\right)$ planes, $\Delta U_{0}=2 U_{0}$ is the velocity difference across the layer and $\rho_{0}$ is the initial gas density. For the cases considered, $\rho_{0}=0.9415 \mathrm{~kg} / \mathrm{m}^{3}, \Delta U_{0}=271.69 \mathrm{~m} / \mathrm{s}$, $\delta_{\omega, 0}=6.859 \times 10^{-3} \mathrm{~m}$, and the convective Mach number $M_{c, 0}$ is 0.35 . The domain size is $0.2 \times 0.22 \times 0.12 \mathrm{~m}^{3}$ and the grid resolution is $288 \times 320 \times 176$ points. The adequacy of the resolution was assessed by computing one-dimensional energy spectra at the transitional times; these plots showed that most of the energy is in the large scales and that there was no accumulation of energy in the small scales, ${ }^{8}$ indicating ap- 
TABLE I. Summary of DNS database. [ $t_{\mathrm{tr}}^{*}$ : Transition time (dimensionless, rounded to nearest $t^{*}$ divisible by 5 ), $t^{*}=t \Delta U_{0} / \delta_{\omega, 0,0} ; \operatorname{Re}_{m}=\rho_{0} \Delta U_{0} \delta_{m} / \mu ; \mathrm{CPU}$ hours are estimates on 64 processors of an SGI Origin 2000.]

\begin{tabular}{lcc}
\hline \hline \multicolumn{1}{c}{ Run } & TP600a2 & TP600a5 \\
\hline $\mathrm{Re}_{0}$ & 600 & 600 \\
$M L_{0}$ & 0.2 & 0.5 \\
$N_{d, 0}$ & 2993630 & 7484075 \\
$t_{\mathrm{tr}}^{*}$ & 105 & 105 \\
$\delta_{m} / \delta_{\omega, 0}$ at $t_{t r}^{*}$ & 2.627 & 2.613 \\
$\mathrm{Re}_{m}$ at $t_{\text {tr }}^{*}$ & 1576 & 1568 \\
$N_{d}$ at $t_{\text {tr }}^{*}$ & 2876604 & 7448608 \\
$\mathrm{CPU}$ hours (estimated) & 2252 & 2981 \\
\hline \hline
\end{tabular}

propriate resolution. At the transition times listed in Table I, the momentum thicknesses $\delta_{m}$ are 2.627 and 2.613 for TP600a2 and TP600a5, respectively.

All thermophysical properties are based on air as the carrier gas and $n$-decane as the drop liquid ${ }^{7}\left(m_{C}\right.$ $=28.97 \mathrm{~kg} / \mathrm{kmol}, \quad m_{V}=142 \mathrm{~kg} / \mathrm{kmol}, \quad \operatorname{Pr}=\mathrm{Sc}=0.697, \quad \rho_{L}$ $\left.=642 \mathrm{~kg} / \mathrm{m}^{3}\right)$. Initially, all the drops have the same temperature $(345 \mathrm{~K})$, which is lower than the initial gas-phase temperature $(375 \mathrm{~K})$ and the liquid boiling temperature $(447.7 \mathrm{~K})$ to promote evaporation. The drop size distribution is specified through the drop Stokes number $\mathrm{St}$ $=\tau_{d} \Delta U_{0} / \delta_{\omega, 0}=d^{2} \rho_{L} \Delta U_{0} / 18 \mu \delta_{\omega, 0}$. In the present study, $\rho_{L}$ and $\mu$ are constant; therefore St is linearly proportional to $d^{2}$. St initially has a Gaussian distribution with mean of 3 and standard deviation of 0.5. Evaporation causes a reduction in the drop size and any drop diminishing to $\mathrm{St} \leqslant 0.1$ is removed from the domain.

Equations (1) and (10) were solved numerically using a fourth-order explicit Runge-Kutta temporal integration for time derivatives and an eighth-order central finite differencing with tenth-order filtering for spatial derivatives; ${ }^{30}$ this filtering introduces a small amount of dissipation that serves only to stabilize the computations for long-time integrations and does not affect the physics of the situation. A fourthorder Lagrange interpolation procedure is used to obtain gasphase variable values at the drop locations. ${ }^{6}$

\section{LES METHODOLOGY}

The LES uses the same mathematical description as the DNS, i.e., Eulerian for the gas phase and Lagrangian for the liquid phase. The LES gas-phase equations are derived by spatially filtering the gas-phase DNS equations [Eq. (1)], and then making various simplifying assumptions. ${ }^{8}$ The filtering operation is defined as

$$
\bar{\psi}(\vec{x})=\int_{V_{f}} \psi(\vec{y}) G(\vec{x}-\vec{y}) d \vec{y},
$$

where $G$ is the filter function and $V_{f}$ is the filtering volume. The present finite-difference computations use a top-hat filter; so $\bar{\psi}$ is simply the volume average. The Favre (densityweighted) filtering is defined as $\tilde{\psi}=\bar{\rho} \psi / \bar{\rho}$. It is assumed that filtering and differentiation commute, which is true except near boundaries (because the size of the filtering volume decreases as the boundary is approached).

The LES uses $N_{\text {cd }}$ computational drops to represent the $N_{d}$ physical drops, that is, each computational drop represents $N_{R} \equiv N_{d} / N_{\text {cd }}$ physical drops. Each LES computational drop belongs to the LES drop field, denoted $\bar{Z}$, and follows the DNS evolution equations [Eq. (10)]:

$$
d \bar{Z} / d t=\sum\left(\tilde{\psi}_{f}, \tilde{\psi}_{s}, \bar{Z}\right)
$$

where $\Sigma$ has the same functional form as in the DNS but is based on $\widetilde{\psi}$ and $\bar{Z}$ instead of $\psi$ and $Z$.

\section{A. Gas-phase LES equations}

The gas-phase LES equations are ${ }^{8}$

$$
\begin{aligned}
& \frac{\partial \bar{\phi}}{\partial t}+\frac{\partial\left(\bar{\phi} \tilde{u}_{j}\right)}{\partial x_{j}}=\frac{\partial}{\partial x_{j}}\left[\Theta_{j}(\bar{\phi})+\Theta_{\mathrm{SGS}, j}\right]+\bar{S}, \\
& \Theta_{\mathrm{SGS}, j}=\left\{0,-\bar{\rho} \tau_{i j},\left(-\bar{\rho} \zeta_{j}-\bar{\rho} \tau_{i j} \tilde{u}_{i}\right),-\bar{\rho} \eta_{j}\right\},
\end{aligned}
$$

where $\bar{\phi}$ denotes the filtered flow field, $\bar{S}=\left\{\bar{S}_{\mathrm{I}}, \bar{S}_{\mathrm{II}, i}, \bar{S}_{\mathrm{III}}, \bar{S}_{I}\right\}$ are the FSTs, $\Theta_{j}$ has the same form as the DNS [Eq. (2)] but is computed on the filtered flow field, and the SGS fluxes are

$$
\tau_{i j}=\widetilde{u_{i} u_{j}}-\tilde{u}_{i} \tilde{u}_{j}, \quad \zeta_{j}=\widetilde{h u_{j}}-\tilde{h} \tilde{u}_{j}, \quad \eta_{j}=\widetilde{Y_{V} u_{j}}-\tilde{Y}_{V} \tilde{u}_{j} .
$$

These equations are based on validated assumptions, ${ }^{8}$ that

$$
\frac{1}{2}\left(\overline{\rho u_{i} u_{i} u_{j}}-\overline{\rho u_{i} u_{i}} \tilde{u}_{j}\right)=\bar{\rho} \tau_{i j} \tilde{u}_{i}
$$

and that $\overline{f(\phi)}=f(\bar{\phi})$, i.e.,

$$
\begin{aligned}
& \tilde{e}=e(\bar{\phi}), \quad \bar{T}=T(\bar{\phi}), \quad \tilde{T}=T(\bar{\phi}), \quad \bar{p}=p(\bar{\phi}), \\
& \tilde{h}=h(\bar{\phi}), \quad \bar{q}_{j}=q_{j}(\bar{\phi}), \quad \bar{j}_{V j}=j_{V j}(\bar{\phi}), \\
& \bar{\sigma}_{i j}=\sigma_{i j}(\bar{\phi}), \quad \overline{u_{i} \sigma_{i j}}=\tilde{u}_{i} \sigma_{i j}(\bar{\phi}),
\end{aligned}
$$

where the listed functions have the same form as in the DNS, and that quantities $\nabla[\overline{f(\phi)}-f(\bar{\phi})]$ are negligible with respect to other terms in the equations. Equation (19) for $\bar{\phi}$ contains terms that cannot be computed directly from $\bar{\phi}$ and that therefore need to be modeled, namely, (1) the FSTs and (2) the SGS fluxes.

\section{B. Models for filtered source terms}

From Eq. (17), the FSTs are properly interpreted by considering a drop located at $\vec{X}$ within the filtering volume $V_{f}$ and its contribution within that volume,

$$
\bar{S}(\vec{x})=\int_{V_{f}} S_{d} \delta(\vec{y}-\vec{X}) G(\vec{x}-\vec{y}) d \vec{y},
$$

where $S_{d} \delta(\vec{y}-\vec{X})$ is the point-source contribution from the drop and $\delta$ is the delta function. When $G$ is a top-hat filter, the exact FSTs are 


$$
\bar{S}=\left(1 / V_{f}\right) \sum_{\alpha=1}^{N_{\alpha}}\left[S_{d}(\psi, Z)\right]_{\alpha},
$$

a volume average over the $N_{\alpha}$ physical drops within the filtering volume $V_{f}$, where $S_{d}$ was defined in Eq. (15). Following Okong'o and Bellan, ${ }^{8}$ the FSTs are modeled from $\bar{\phi}$ and $\bar{Z}$ as

$$
\bar{S}=N_{R} \sum_{\beta=1}^{N_{\beta}}\left(1 / V_{f}\right)\left[S_{d}\left(\tilde{\psi}(\bar{\phi}), \bar{Z}\left(N_{R}\right)\right)\right]_{\beta},
$$

where the summation is over the $N_{\beta}$ computational drops within the filtering volume $V_{f}$, and $S_{d}$ has the same functional form as in the DNS [Eq. (15)] with $\tilde{\psi}$ being the model for $\psi$, that is, without modeling direct SGS effects on drop evolution. On an a priori basis, the model devoid of direct SGS effects on drop evolution proved almost as good as a model accounting deterministically for direct SGS effects and superior to a model accounting statistically for such effects. ${ }^{8}$ Following the LES framework, simpler, less computationally intensive models are first tried to evaluate their potential before considering more computationally intensive models; the present study represents the first step in this endeavor.

\section{Subgrid-scale flux models}

For compactness of notation, the SGS fluxes for a variable $\varphi$ are denoted as

$$
\theta_{j}(\varphi)=\left(\widetilde{\varphi u_{j}}-\tilde{\varphi} \widetilde{u}_{j}\right)
$$

where $\theta_{j}\left(u_{i}\right)=\tau_{i j}, \theta_{j}\left(Y_{V}\right)=\eta_{j}$ and $\theta_{j}(h)=\zeta_{j}$. The model for $\theta_{j}(\varphi)$, to be calculated on the filtered flow field $(\bar{\phi})$, is denoted $\mathcal{M}_{j}(\widetilde{\varphi} ; \bar{\phi}, \bar{\Delta})$ (associated with the filter width $\bar{\Delta}$ and with the velocity $\left.\widetilde{u}_{j}\right) ; \mathcal{M}_{j}$ does not contain the model coefficient. For the SGS fluxes defined in Eq. (20), the three typical SGS models are considered here: The Smagorinsky ${ }^{31}$ (SM) model combined with the Yoshizawa ${ }^{32}$ (YO) model is

$$
\begin{aligned}
& \theta_{j}\left(u_{i}\right)=C_{\mathrm{SM}} \mathcal{M}_{j}\left(\widetilde{u}_{i}\right)+C_{\mathrm{YO}} \mathcal{M} \delta_{i j} / 3, \\
& \mathcal{M}(\bar{\phi}, \bar{\Delta})=\bar{\Delta}^{2} S^{2}(\bar{\phi}), \\
& \mathcal{M}_{j}\left(\widetilde{u_{i}} ; \bar{\phi}, \bar{\Delta}\right)=-\bar{\Delta}^{2} S(\bar{\phi})\left[S_{i j}(\bar{\phi})-S_{k k}(\bar{\phi}) \delta_{i j} / 3\right], \\
& \theta_{j}(\varphi)=C_{\mathrm{SM}} \mathcal{M}_{j}(\widetilde{\varphi}), \quad \varphi=Y_{V}, h, \\
& \mathcal{M}_{j}(\widetilde{\varphi} ; \bar{\phi}, \bar{\Delta})=-\bar{\Delta}^{2} S(\bar{\phi}) \frac{1}{2} \frac{\partial \widetilde{\varphi}}{\partial x_{j}},
\end{aligned}
$$

where $S^{2}=S_{i j} S_{i j}$. The gradient (GR) model is ${ }^{33}$

$$
\begin{aligned}
& \theta_{j}(\varphi)=C_{\mathrm{GR}} \mathcal{M}_{j}(\widetilde{\varphi}), \\
& \mathcal{M}_{j}(\widetilde{\varphi} ; \bar{\phi}, \bar{\Delta})=\bar{\Delta}^{2} \frac{\partial \widetilde{\varphi}}{\partial x_{k}} \frac{\partial \widetilde{u}_{j}}{\partial x_{k}}, \quad \varphi=u_{i}, Y_{V}, h .
\end{aligned}
$$

The scale-similarity (SS) model is ${ }^{34}$

$$
\begin{aligned}
& \theta_{j}(\varphi)=C_{\mathrm{SS}} \mathcal{M}_{j}(\widetilde{\varphi}), \\
& \mathcal{M}_{j}(\tilde{\varphi} ; \bar{\phi}, \bar{\Delta})=\left(\widehat{\widetilde{\varphi} \tilde{u}_{j}}-\hat{\tilde{\varphi}} \hat{\tilde{u}}_{j}\right), \quad \varphi=u_{i}, Y_{V}, h,
\end{aligned}
$$

where the overhat $\left({ }^{\wedge}\right)$ denotes (unweighted) filtering at the test-filter level $\hat{\Delta} \geqslant \bar{\Delta}$.

For the SS model, the DNS-calibrated constantcoefficient value ${ }^{8}$ of $C_{\mathrm{SS}}=1.996$ (model denoted SSC; $\hat{\Delta}=\bar{\Delta}$ ) is used here. For the GR and SM models, the calibrated constant coefficients, ${ }^{8}$ while adequate for SP flows were not consistently stable for TP flows. Therefore, dynamic Smagorinsky (SMD) and dynamic gradient (GRD) models were considered, where the coefficients are dynamically computed as part of the LES solution. Basically, dynamic modeling attempts to deduce the SGS behavior from that of the smallest resolved scales in LES. The essence of the method is to relate the grid-level SGS flux $\theta_{j}$ and the test-level SGS flux,

$$
T_{j}(\varphi)=\left(\widehat{\varphi u_{j}}-\hat{\tilde{\varphi}} \hat{\tilde{u}}_{j}\right),
$$

to the test-level resolved flux $L_{j}$ through the Germano identity: ${ }^{35}$

$$
L_{j}(\varphi) \equiv \widehat{\widetilde{\varphi} \widetilde{u}_{j}}-\hat{\varphi} \hat{\bar{u}}_{j}=T_{j}-\hat{\theta}_{j} .
$$

Using a $T_{j}$ model having the same functional form as the $\theta_{j}$ model but based on $\hat{\tilde{\varphi}}$ and $\hat{\bar{\Delta}}$ (instead of $\tilde{\varphi}$ and $\bar{\Delta}$ ), the $L_{j}$ model is

$$
M_{j}(\varphi)=\mathcal{M}_{j}(\hat{\tilde{\varphi}})-\widehat{\mathcal{M}}(\widetilde{\varphi}) .
$$

The filter width $\hat{\bar{\Delta}}$ due to the refiltering is $\hat{\bar{\Delta}}^{2}=\bar{\Delta}^{2}+\hat{\Delta}^{2}$ for the top-hat filter. ${ }^{36}$

The domain-average model coefficient is computed using a least-squares fit, ${ }^{37}$

$$
C(\varphi)=\frac{\left\langle\left\langle L_{j}(\varphi) M_{j}(\varphi)\right\rangle\right\rangle}{\left\langle\left\langle M_{k}(\varphi) M_{k}(\varphi)\right\rangle\right\rangle},
$$

where $\langle\langle\rangle\rangle$ denotes averaging over the entire domain; the coefficient can also be computed on homogeneous planes by replacing the domain averaging with plane averaging. The coefficients were obtained using domain averaging for $\tau_{i j}$, domain or homogeneous-plane averaging for $\zeta_{j}$ (respectively, for the SMD or GRD model) and homogeneous-plane averaging for $\eta_{j}$. These averagings were consistently stable for SP and TP flows. All the dynamic models use $\hat{\Delta}=2 \bar{\Delta}$. Three coefficients were utilized for SMD (for $\tau_{i j}, \zeta_{j}$ and $\eta_{j}$ ), and four coefficients were employed for GRD (for $\tau_{i j, i=j}, \tau_{i j, i \neq j}, \zeta_{j}$, and $\eta_{j}$ ). (The use of dimensional variables in the present formulation necessitates the separate computation of the coefficient for each type of SGS flux.) In view of the observations of Okong'o and Bellan ${ }^{8}$ that the SS and GR models have much better correlations with the SGS fluxes than does the SM model, no 'mixed' models [in which the (dynamic) SM model is used to add dissipation to the other two models] were considered. 
TABLE II. FC-DNS and LES corresponding to DNS cases listed in Table I; $N_{R}=8\left(\delta_{m} / \delta_{\omega, 0}\right.$ and $N_{\mathrm{cd}}$ at transition time $t_{\mathrm{tr}}^{*}=105$; CPU hours on SGI Origin 2000).

\begin{tabular}{|c|c|c|c|c|c|c|c|c|}
\hline \multirow[b]{2}{*}{$\begin{array}{l}\text { SGS } \\
\text { Model }\end{array}$} & \multicolumn{4}{|c|}{ TP600a2; $N_{\mathrm{cd}, 0}=374189$} & \multicolumn{4}{|c|}{ TP600a5; $N_{\mathrm{cd}, 0}=935498$} \\
\hline & $\delta_{m} / \delta_{\omega, 0}$ & $N_{\mathrm{cd}}$ & $N_{\mathrm{cd}}$ within layer & $\begin{array}{c}\mathrm{CPU} \\
\text { (h) }\end{array}$ & $\delta_{m} / \delta_{\omega, 0}$ & $N_{\text {cd }}$ & $N_{\mathrm{cd}}$ within layer & $\begin{array}{c}\mathrm{CPU} \\
\text { (h) }\end{array}$ \\
\hline FC-DNS & 2.631 & 359550 & 200510 & $\cdots$ & 2.617 & 931048 & 507595 & $\cdots$ \\
\hline GRD & 2.102 & 358377 & 227931 & 20 & 2.313 & 931462 & 529789 & 37 \\
\hline SMD & 2.223 & 351836 & 163746 & 20 & 2.230 & 929271 & 455615 & 37 \\
\hline SSC & 2.270 & 351140 & 183016 & 19 & 2.152 & 929275 & 381218 & 38 \\
\hline
\end{tabular}

\section{Initial conditions and numerical procedure}

As stated in Sec. I, the best that LES results can achieve is to reproduce the FC-DNS. The choice is here to study LES conducted with $N_{\text {cd }}$ computational drops selected from the array of $N_{d}$ physical drops using a stride of $N_{R}$. Therefore, it is appropriate to use for LES the FC-DNS initial condition, where a subset of the DNS drop field was extracted using the same $N_{R}$ value. The type of LES filter, here a cubic top-hat filter, explicitly appears in computing the initial condition and also during the LES when computing the SGS fluxes (if the similarity or a dynamic SGS model is used) and the FSTs. The DNS cases considered here are listed in Table I; the corresponding LES cases are listed in Table II. The LES grid is coarser than the DNS grid, with $\Delta x_{\mathrm{LES}}=4 \Delta x_{\mathrm{DNS}}$ (72 $\times 80 \times 44$ points) and $\bar{\Delta}=2 \Delta x_{\mathrm{LES}}$; also, the choice here is $N_{R}=8$. The LESs are performed using the same numerical scheme as the DNS (see Sec. II D). Most of the analysis is performed at the DNS transitional times $t_{\text {tr }}^{*}$, listed in Table I. Since all LESs use here the same FST model, they will be identified according to the SGS-flux model as GRD, SMD, or SSC LES.

At the DNS transition time, the FC-DNS $\delta_{m}$ is almost the same as the DNS (within $0.2 \%$ ) but the LES $\delta_{m}$ are $11 \%-$ $15 \%$ lower than the DNS value, with the SSC (GRD) being closest to the DNS for TP600a2 (TP600a5). The LESs have approximately the same total number of drops as the FCDNS (98\%-100\%); however, they have too many (SMD; $105 \%-115 \%$ ) or too few (GRD, SSC; 75\%-91\%) drops within the layer, with the layer edge planes defined where $\left\langle u_{1}\right\rangle$ is $1 \%$ of the free-stream values. Given the considerably lower CPU requirements for LES compared to DNS (LES reduced CPU-time by a factor of 113 for TP600a 2 and 78 for TP600a5 compared to DNS), the interest is whether the detailed characteristics of the layer are accurately predicted by LES. Because in combustion processes ignition is through chemical reactions that occur locally, it is the reproduction of these detailed mixing characteristics that is of interest for further combustion.

\section{DETAILED CHARACTERISTICS}

The rationale of the study is to follow an increasing depth of inquiry regarding the LES capability. At the minimum, LES must predict well the flow dynamic characteristics. Not only does this mean that the gas flow will be well predicted, but since it is well known that drops accumulate in regions of low vorticity and high strain, ${ }^{38}$ success with the dynamics indicates that the drop spatial distribution will also be well predicted; this effect was indeed shown in a posteriori studies where failure of the drops to be at the correct FC-DNS location was directly attributed to poor LES predictions of the flow dynamics. ${ }^{22}$ If the drop spatial distribution is correct and if the FST model accurately portrays the drop/ flow interactions, it is expected that mixing of air and vapor resulting from drop evaporation will also be well predicted; it is this mixture that is of paramount interest for combustion applications and will be the subject of the present scrutiny. Finally, as an issue of scientific interest, it is intriguing to examine to what extent LES can reproduce the flow dissipation.

\section{A. Dynamic characteristics}

To assess the quality of LES in predicting the dynamic characteristics, illustrated in Fig. 2 is the time evolution of the enstrophy $\langle\langle\omega \cdot \omega\rangle\rangle$, a measure of stretching and tilting which promotes the generation of turbulence. For convenience, the bar and tilde that denote filtered quantities are omitted; all quantities are computed from the filtered flow field quantities. For both TP600a2 and TP600a5, $\langle\langle\omega \cdot \omega\rangle\rangle$ increases dramatically at roll-up, grows steadily, with a slight dip for TP600a2 at the first pairing, culminates shortly after the second pairing, and declines afterwards. Considering the LES, the SMD model has much less vorticity generation than the FC-DNS, with only a slight increase during the layer evolution. In contrast, both the GRD and SSC models qualitatively mimic the FC-DNS $\langle\langle\omega \cdot \omega\rangle\rangle$ evolution, with the GRD being a better quantitative match. Both GRD and SSC peak at a lower value and at an earlier time than the FCDNS; however, the GRD is closer to the FC-DNS. The inability of the GRD and SSC models to fully replicate the FC DNS enstrophy in LES shows that the SGS-flux models are not a perfect palliate for the removed small-scale information. The indications are that the SMD model has even much less small-scale activity and generation than the other two LES models.

The local enstrophy in the between-the-braid plane $\left(x_{3} / \delta_{\omega, 0}=8.75\right)$ is plotted in Fig. 3 for the TP600a2 FC-DNS and LES at the DNS transition time $t^{*}=105$ (results for TP600a5 are similar). The contour levels are from $10 \%$ to $90 \%$ of the maximum value of $\omega \cdot \omega$ in the plane. The FCDNS shows substantial small-scale activity, well distributed 


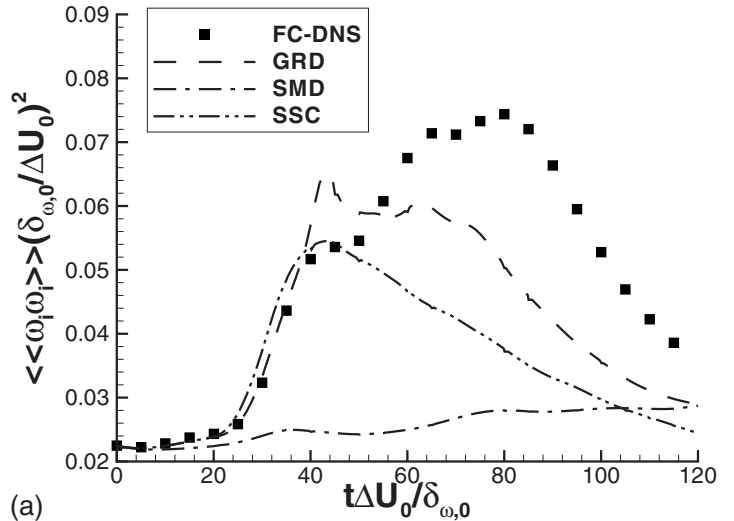

(a)

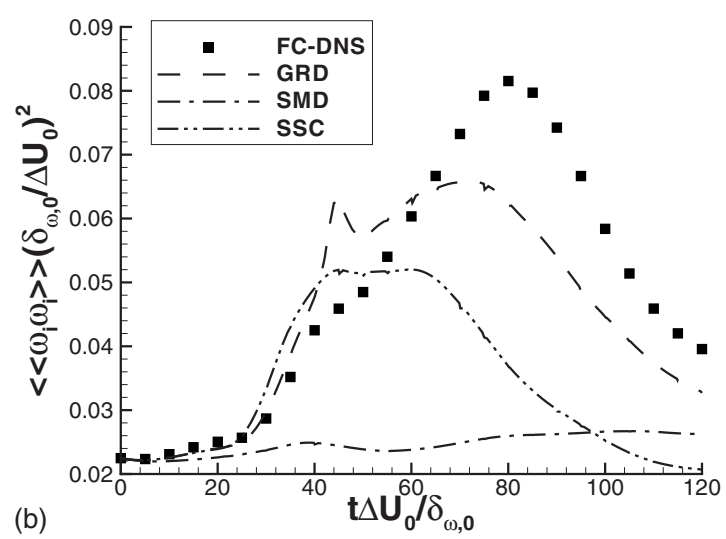

FIG. 2. Evolution of domain-averaged enstrophy for (a) TP600a2 and (b) TP600a5.

(a)

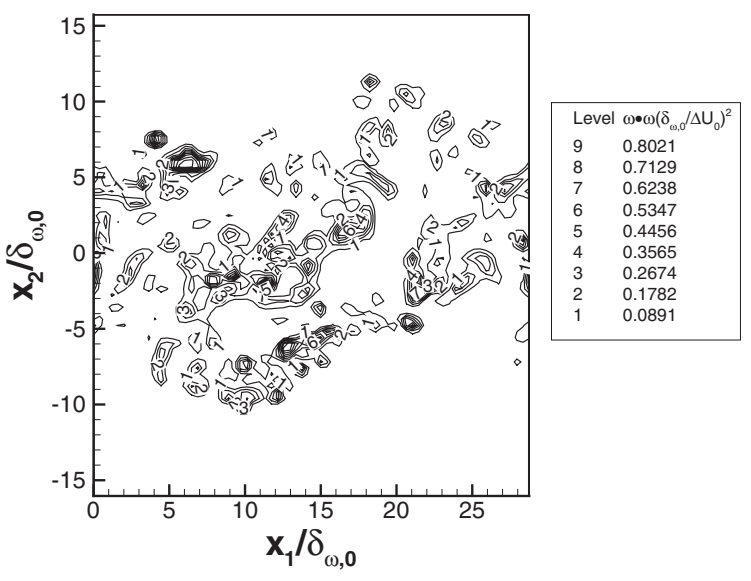

(b)

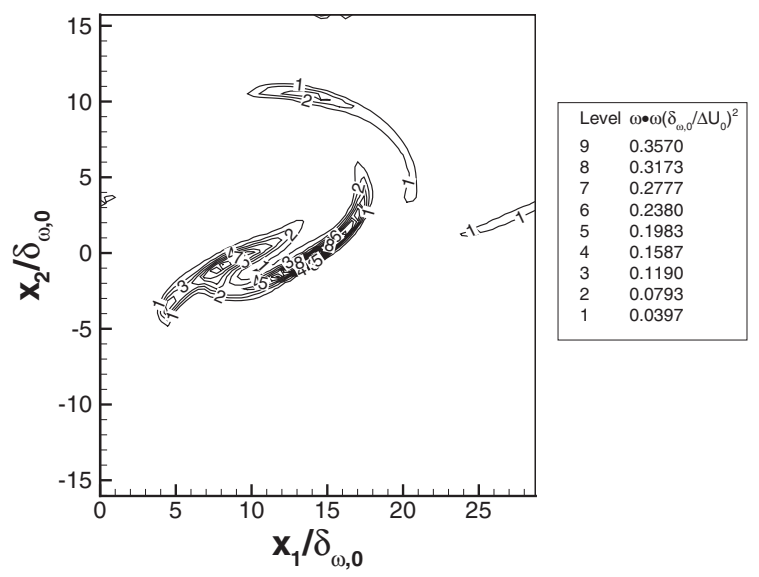

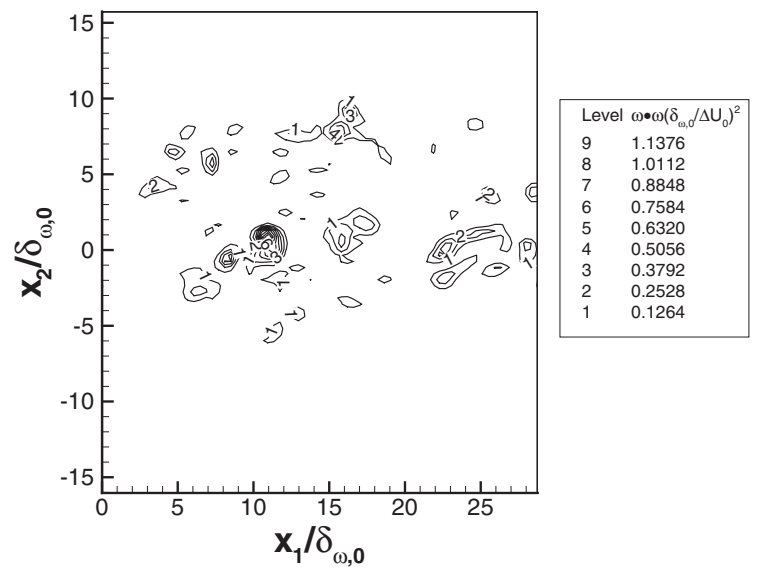

within the ultimate vortex which results from the pairing of the four initial spanwise vortices. The SMD LES displays only enstrophy associated with the largest-scale vortex and none associated with small-scale structures, confirming the indications of the global measures in Fig. 2 that the SMD has little generation of small-scale structures. Furthermore, as in the global measures, the SMD enstrophy level is much lower than the FC-DNS. The GRD and SSC models show smallscale structures within the ultimate vortex, similar to the FCDNS; however, fewer structures are observed due to the lower resolution of the LES compared to the DNS from which the FC-DNS has been extracted. In this plane, the FC-DNS range of values is better matched by the SSC than by the GRD, although the size and distribution of small structures of the GRD more resemble the FC-DNS than does the SSC.

The enstrophy generation at the DNS transition time is analyzed by means of the budget:

$$
\begin{aligned}
D(\omega \cdot \omega) / D t= & 2 \omega \cdot[(\omega \cdot \nabla) \mathbf{u}]-2(\omega \cdot \omega)(\nabla \cdot \mathbf{u}) \\
& +2 \omega \cdot\{\nabla \times[(-\nabla p+\nabla \cdot \sigma) / \rho]\} \\
& +2 \omega \cdot\left\{\nabla \times\left[\left(\mathbf{S}_{\mathrm{II}}-\mathbf{u} S_{\mathrm{I}}\right) / \rho\right]\right\} .
\end{aligned}
$$

Averages in homogeneous planes of $D(\omega \cdot \omega) / D t$, plotted in Fig. 4 for TP600a2 and TP600a5, show for TP600a2 approximately the same total $D(\omega \cdot \omega) / D t$ for all LESs, which

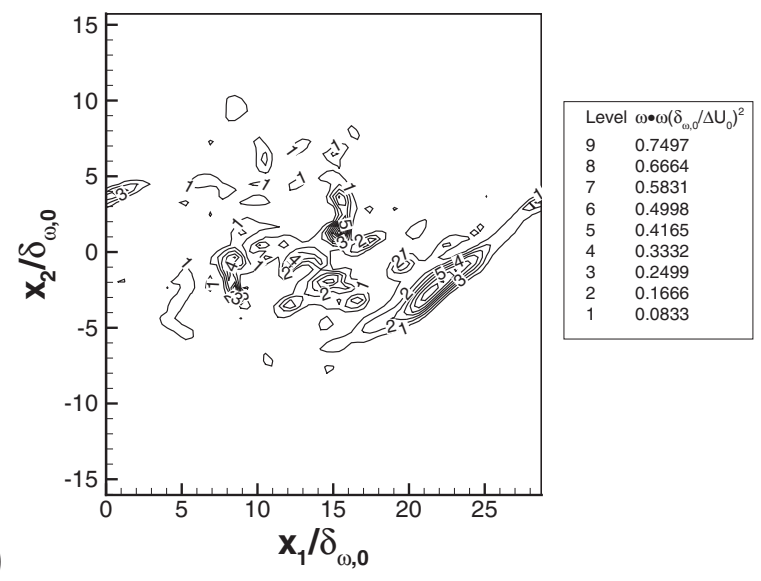

FIG. 3. Enstrophy, TP600a2 at $t^{*}=105$, between-the-braid plane: (a) FC-DNS, (b) GRD, (c) SMD, and (d) SSC. 


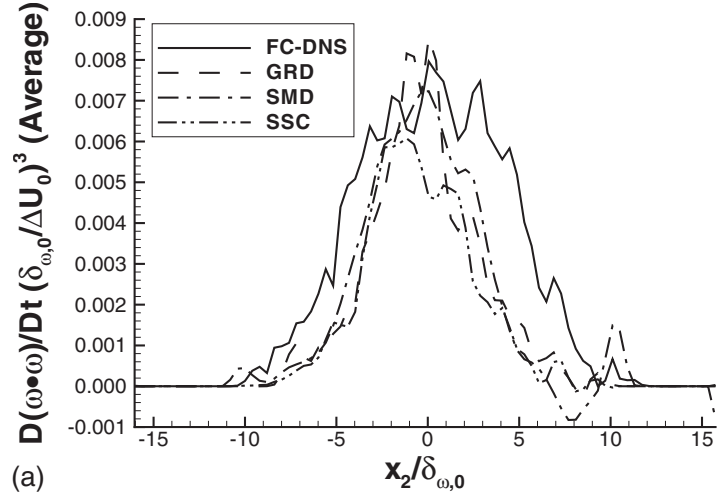

(a)

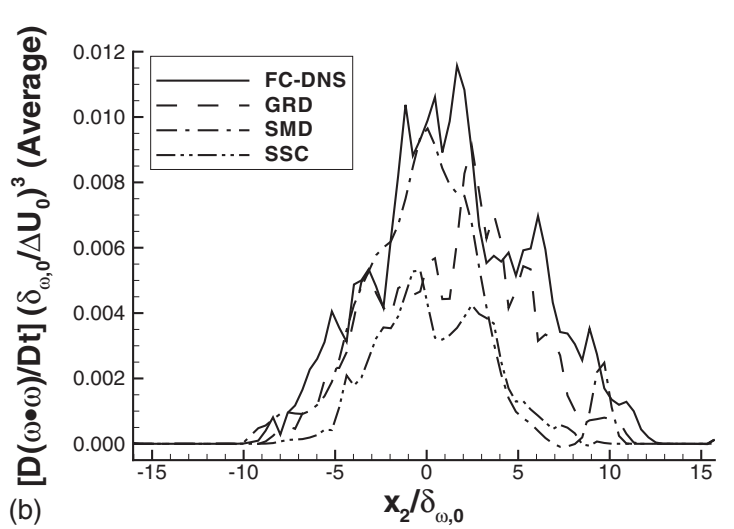

FIG. 4. Homogeneous-plane enstrophy averages at $t^{*}=105$ : (a) TP600a2 and (b) TP600a5.

compares well to the FC-DNS. For TP600a5, the results are similar, although the SSC model has a smaller magnitude of $D(\omega \cdot \omega) / D t$ than the other LESs. All LES models seem to capture the spatial extent of vorticity activity, signifying that the extent is mainly governed by the large-scale structure, independent of the SGS model. However, differences emerge in the relative contributions of the six terms in Eq. (40), plotted in Fig. 5 for TP600a2 FC-DNS and LES. All models follow the FC-DNS in that the stretching/tilting term has the largest magnitude of terms that are on average positive, while the viscous term has the largest magnitude of negative terms. Closer examination of the SMD results shows an overall lower magnitude of stretching/tilting and viscous terms compared to the FC-DNS while having the largest magnitude of source terms. Also, the SMD has a dip in the stretching/tilting term in the upper stream that does not appear in the FC-DNS or other LES models.

The assessment of the LES dynamic characteristics is that the SMD model does not capture small-scale activity, while the GRD and SSC models do so to a great extent. Large-scale activity, such as the extent of the ultimate vortex, appears to be independent of the SGS model, and thus is captured by all LES.

\section{B. Drop distribution}

The assessment of the drop distribution prediction evaluates the coupled effect of the dynamics and FST model. Of note is that LES do not incorporate here direct SGS effects on the drops, but SGS effects do enter indirectly in affecting the resolved flow field that is felt by the drops. Here the emphasis is in whether the LES preserve the relationship between dynamics and drop locations.

The drop number density, $\rho_{n}=N_{R}\left(N_{\beta} / V_{f}\right)$ where $N_{\beta}$ is the number of computational drops in the filtering volume $V_{f}$, is plotted in Fig. 6 for TP600a2 in the between-the-braid plane. For $\rho_{n}$, the FC-DNS template can be considered a statistical sample of the DNS field. The drops in the SMD are incorrectly located, being concentrated on the edges of the ultimate vortex, rather than within it. The GRD and SSC drops, on the other hand, are distributed similarly to the FCDNS, with voids corresponding to high vorticity regions (see Fig. 3), although their maximum $\rho_{n}$ is about $30 \%$ lower compared to the FC-DNS. The number of drops within the layer versus St for the FC-DNS and the LES is shown in Fig. 7. The range of St values and the St with the largest number of drops seem well predicted by all models. However, the TP600a2 SMD has too many drops for $\mathrm{St}>0.7$ and TP600a5 SMD has too many drops for $\mathrm{St}>1.7$. The TP600a2 GRD and SSC have too few drops for $\mathrm{St}>0.9$ and $\mathrm{St}>1.1$, respectively; the TP600a5 GRD and SSC have too few drops for $\mathrm{St}>1.4$.

The drop distribution conditioned on the second invariant of the deformation tensor

$$
I_{2}(\mathbf{D})=-S_{i j} S_{i j} / 2+S_{i i} S_{j j} / 2+\omega_{i} \omega_{i} / 4,
$$

where $D_{I j}=\partial u_{I} / \partial x_{j}$, reveals the distribution of drops in rotational or compressible regions $\left[I_{2}(\mathbf{D})>0\right]$ compared to the strain-dominated regions $\left[I_{2}(\mathbf{D})<0\right]$. For these layers, $S_{i i} S_{j j} / 2$ is much smaller than the other terms, so $I_{2}(\mathbf{D})>0$ regions are vorticity dominated. The number of drops with particular values of $I_{2}(\mathbf{D})$ is plotted in Fig. 8; the value of $I_{2}(\mathbf{D})$ associated with each drop is obtained by interpolating $I_{2}$ (D) from the grid points to the drop locations using fourthorder Lagrangian interpolation. Qualitatively, all LESs match the DNS in that drops are most likely to be in regions of $I_{2}(\mathbf{D}) \simeq 0$; the preponderance of drops in the $I_{2}(\mathbf{D}) \simeq 0$ regions indicates that most drops are still in the lower stream part of the mixing layer where there is neither strain nor vorticity. However, the number of drops is reduced for all LESs when compared to the FC-DNS. For TP600a5, the reduction is consistent whether $I_{2}(\mathbf{D})$ is positive or negative, with the GRD best matching the FC-DNS, followed by the SSC, and worst agreement from the SMD. For TP600a2, the worst result still comes from the SMD model, but the GRD and SSC models now perform comparably, and on the positive $I_{2}(\mathbf{D})$ side they match the FC-DNS very well. The lower mass loading leads to less vapor saturation and more evaporation, and thus smaller drops for the TP600a2; these drops tend to better follow the flow than the larger TP600a5 drops and are therefore less susceptible to modeling errors.

The drop number density is plotted in Fig. 9, conditionally domain averaged on $I_{2}(\mathbf{D})$. The FC-DNS $\rho_{n}$ average is highest for $I_{2}(\mathbf{D}) \simeq 0$, followed by $I_{2}(\mathbf{D})<0$, and lowest for $I_{2}(\mathbf{D})>0$. The GRD model best matches the FC-DNS curve, with good agreement throughout the $I_{2}(\mathbf{D})$ range. At the other extreme, the SMD model has a truncated $I_{2}(\mathbf{D})$ range, 


\begin{tabular}{|c|c|c|}
\hline 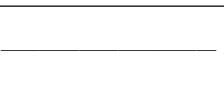 & $2 \omega \cdot[(\omega \cdot \nabla) \mathbf{u}]\left(\delta_{\omega, 0} / \Delta U_{0}\right)^{3}$ & $\ldots \ldots \ldots 2 \omega \cdot\{\nabla \times[(\nabla \cdot \sigma) / \rho]\}\left(\delta_{\omega, 0} / \Delta U_{0}\right)^{3}$ \\
\hline$-\cdot-\cdot-\cdot-$ & $-2(\omega \cdot \omega)(\nabla \cdot \mathbf{u})\left(\delta_{\omega, 0} / \Delta U_{0}\right)^{3}$ & $---2 \omega \cdot\left[\nabla \times\left(\mathbf{S}_{I I, i} / \rho\right)\right]\left(\delta_{\omega, 0} / \Delta U_{0}\right)^{3}$ \\
\hline$-\cdots-\cdots-$ & $-2 \omega \cdot[\nabla \times(\nabla p / \rho)]\left(\delta_{\omega, 0} / \Delta U_{0}\right)^{3}$ & $-2 \omega \cdot\left[\nabla \times\left(\mathbf{u} S_{I} / \rho\right)\right]\left(\delta_{\omega, 0} / \Delta U_{0}\right)^{3}$ \\
\hline
\end{tabular}

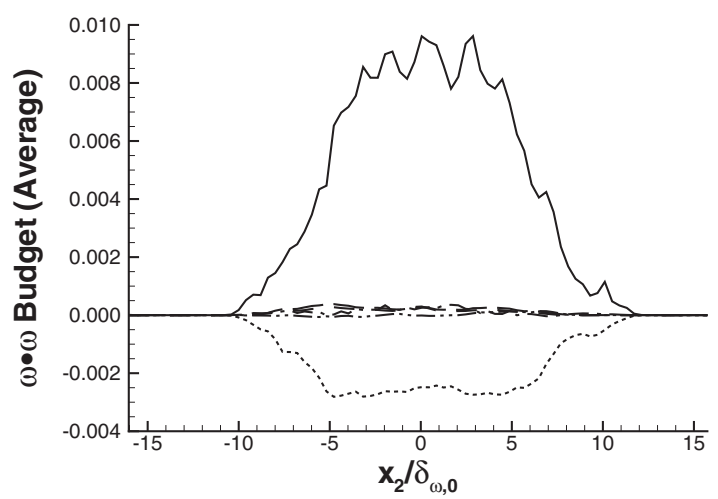

(a)

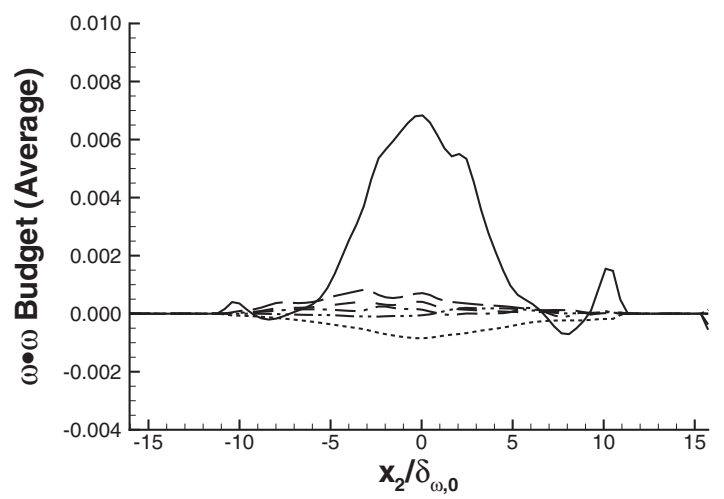

(b)

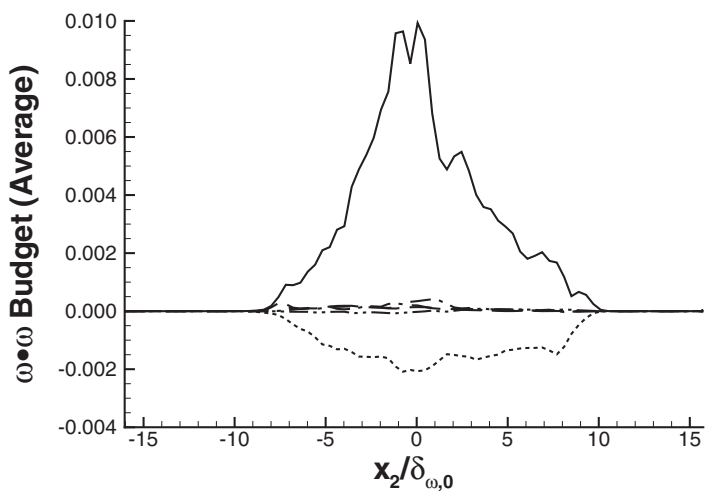

(d)

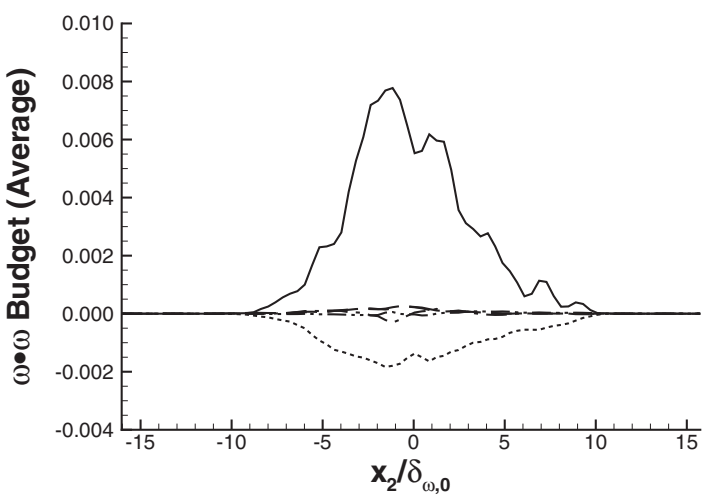

FIG. 5. Enstrophy budget, TP600a2 at $t^{*}=105$, averages in homogeneous planes: (a) FC-DNS, (b) GRD, (c) SMD, and (d) SSC.

particularly on the negative side, with the highest number density for $I_{2}(\mathbf{D})\left(\delta_{\omega, 0} / \Delta U_{0}\right)^{2} \simeq-0.022$; both the location and magnitude of the $\rho_{n}$ peak differ markedly from the FC-DNS. The SSC model, while not as good as the GRD, is much better than the SMD, although for TP600a2 (TP600a5) it overpredicts (underpredicts) the $\rho_{n}$ average for $I_{2}(\mathbf{D})<0$.

Therefore, the range of drop sizes is well predicted by all SGS models. However, not only is the flow field, especially the small-scale structures, not well predicted by the SMD model, but the SMD model does not predict the correct relationship between flow structures and drop distributions. This serves as further confirmation of the superiority of the GRD and SSC models over the SMD model.

\section{Mixing characteristics}

Mixing determines the gaseous composition which together with the temperature governs the propensity for combustion. Prediction of the mixing characteristics is the coupled result of flow dynamics and drop distribution. Mixing of fuel and oxidizer is best quantified by the equivalence ratio. The local and global equivalence ratios can be computed as

$$
\Phi=\frac{\left(Y_{V} / Y_{C}\right)}{\left(Y_{V} / Y_{C}\right)_{\mathrm{st}}}, \quad \Phi_{g}=\frac{\left(M_{V} / M_{C}\right)}{\left(M_{V} / M_{C}\right)_{\mathrm{st}}},
$$

where $M_{V}$ and $M_{C}$ are the total mass of vapor and carrier gas, respectively, in the domain and subscript st indicates the stoichiometric conditions for the carrier gas and vapor reaction.

To assess the species mixing, the evolution of the global equivalence ratio is depicted in Fig. 10 for both TP600a2 and TP600a5. All LESs show good agreement with the FC-DNS, and thus have the proper global amount of drop evaporation, suggesting that in this flow configuration the global evaporation is mainly driven by large-scale structures. The local equivalence ratio is plotted in Fig. 11 for TP600a2 in the between-the-braid plane. The contour plots in Fig. 11 show that all models do well in capturing the range of values of $\Phi$. However, the SMD model does not show appropriate smallscale activity, while the GRD and SSC capture this aspect.

The temperature is plotted in Fig. 12 also for TP600a2 in the between-the-braid plane. The local temperature depends on the histories of convective effects, heat flux, and drop number density. The temperature variation observed in the FC-DNS is mainly due to the cooling effect of the drops, 
(a)
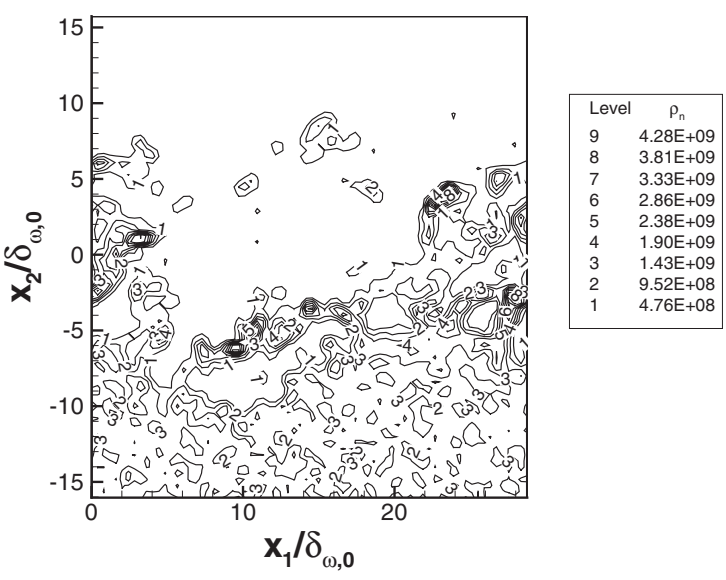

(c)
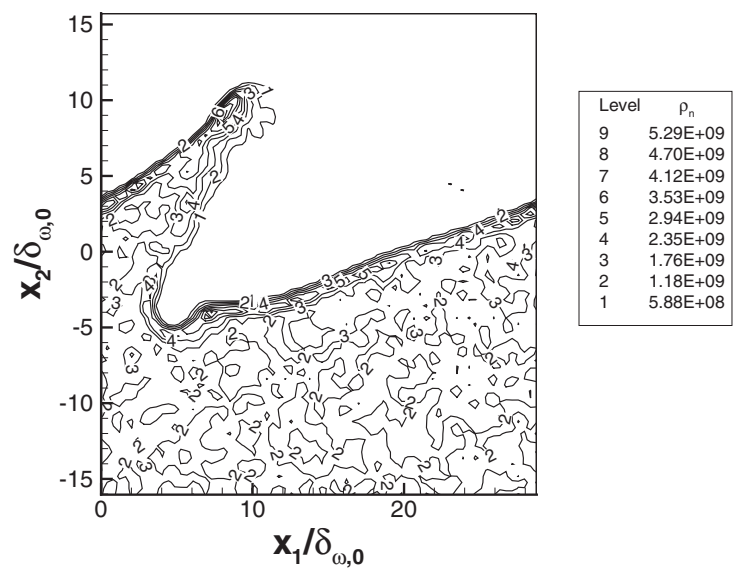
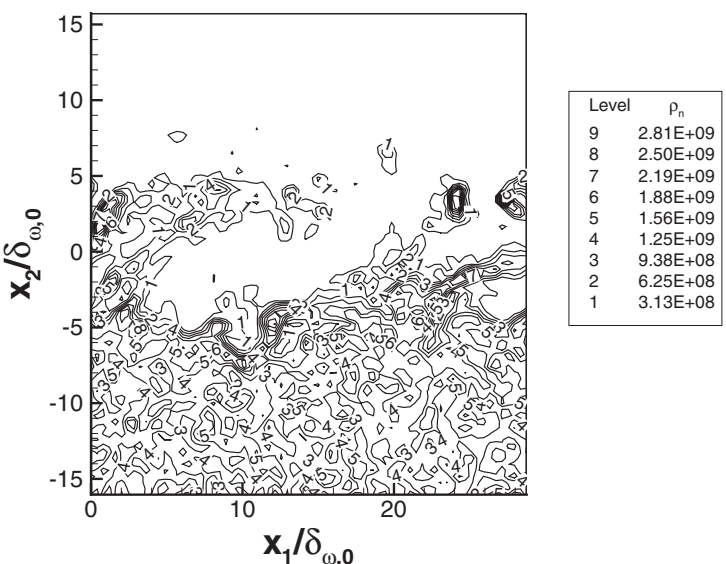

(b)

(d)

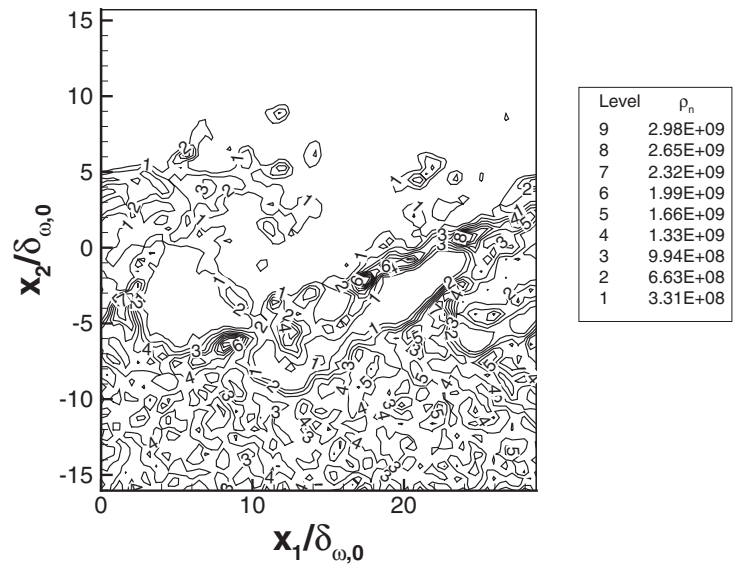

FIG. 6. Drop number density $\left(\mathrm{m}^{-3}\right)$, TP600a2 at $t^{*}=105$, between-the-braid plane: (a) FC-DNS, (b) GRD, (c) SMD, and (d) SSC.

which have reduced the carrier gas temperature from the free-stream $375 \mathrm{~K}$ value. For GRD and SMD in the lower stream, the temperature has fallen below the initial drop temperature of $340 \mathrm{~K}$, but this did not occur for the SSC model. Therefore, the small reduction in the gas temperature below the initial drop temperature is an indication that the approximations in the SGS model rather than FST model is marginally deficient; moreover, because the SGS flux is negligible in the free-stream region, we can attribute this discrepancy to inaccuracies in the pressure/temperature relationship through the equation of state. In addition to the large-scale temperature variation, most noticeable in the cross-stream direction, there are small-scale variations on the order of small-scale structures observed in $\omega \cdot \omega$ (Fig. 3). Comparing the FC-DNS to the LES, it is clear that the SMD model, as in the dynamics, does not capture the small-scale variations. Also, the SMD temperature range exceeds that of the FC-DNS. The SSC model best matches the FC-DNS temperature range, while the GRD leads to a slightly colder flow field. Both the GRD and SSC show the small-scale variations observed in the FC-DNS.

In view of the poor local performance of the SMD model, global results must be interpreted with caution when used as indicators of small-scale or turbulence activity. Since it is the local thermodynamic state that governs combustion,
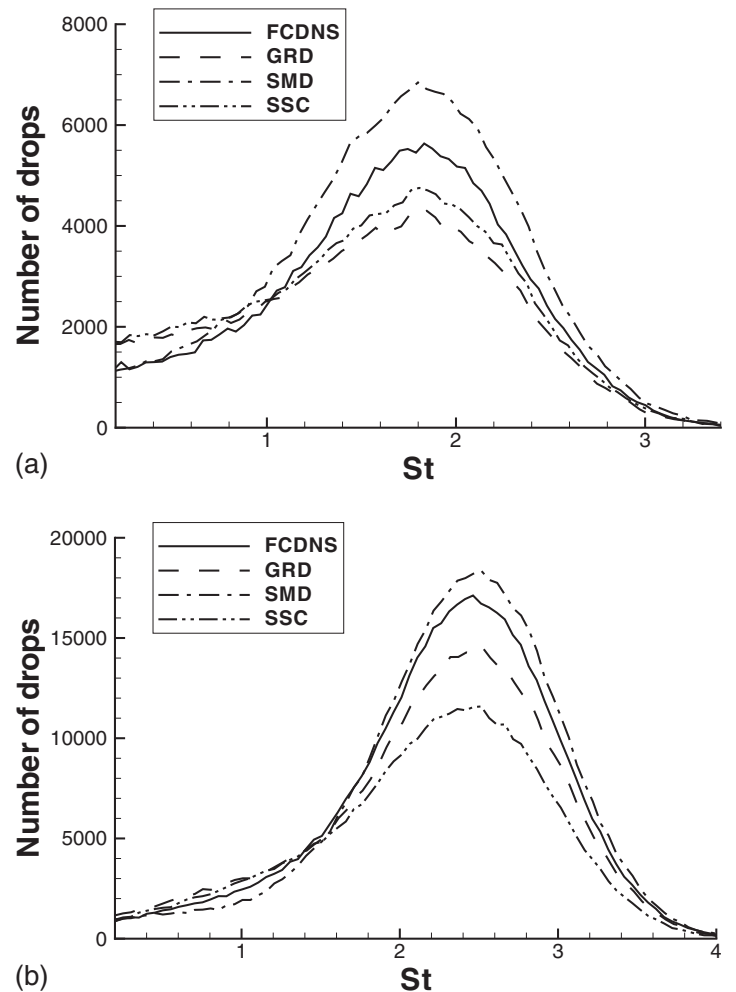

FIG. 7. Number of drops within layer vs Stokes number: (a) TP600a2 and (b) TP600a5. 


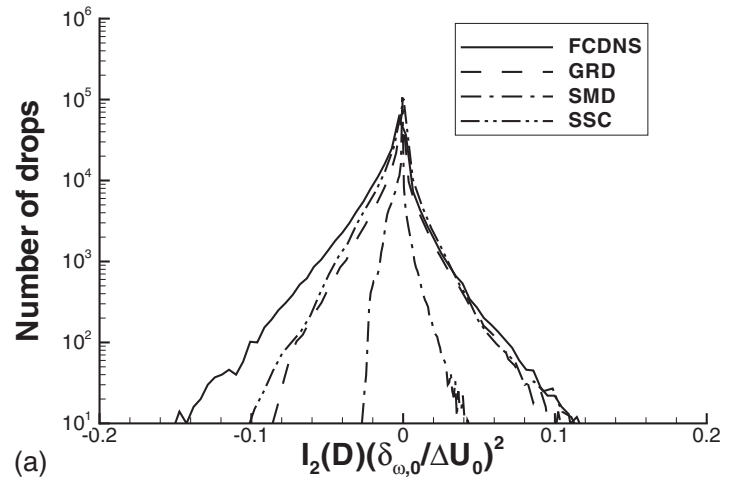

(a)

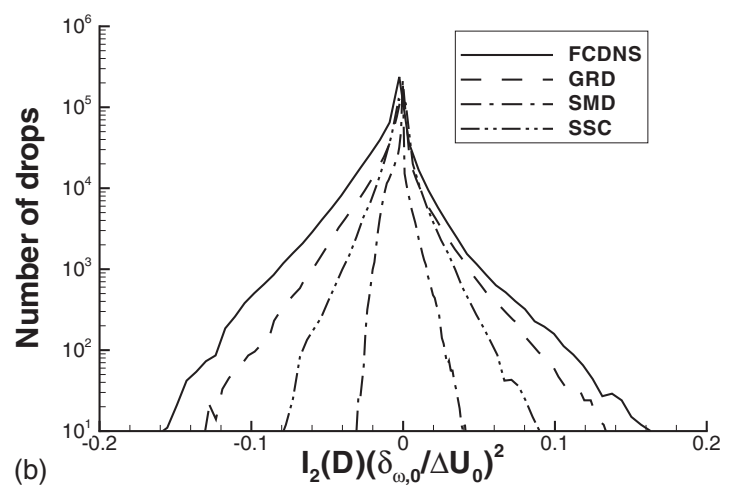

FIG. 8. Number of drops within layer vs $I_{2}(\mathbf{D})$ : (a) TP600a2 and (b) TP600a5.

including pollutant prediction such as soot, the poor local predictions of the SMD model seem to disqualify it from combustion applications.

\section{Irreversible entropy production}

The irreversible entropy production is the dissipation, which is of crucial importance in determining the characteristics of turbulent flows because it contains the viscous dissipation which measures the loss of mechanical energy to heat, the scalar dissipation which manifests in the mixing, as well as the dissipation due to temperature gradients and that due to drop source terms. The effect of the LES dynamic, drop distribution, and mixing characteristics on duplicating
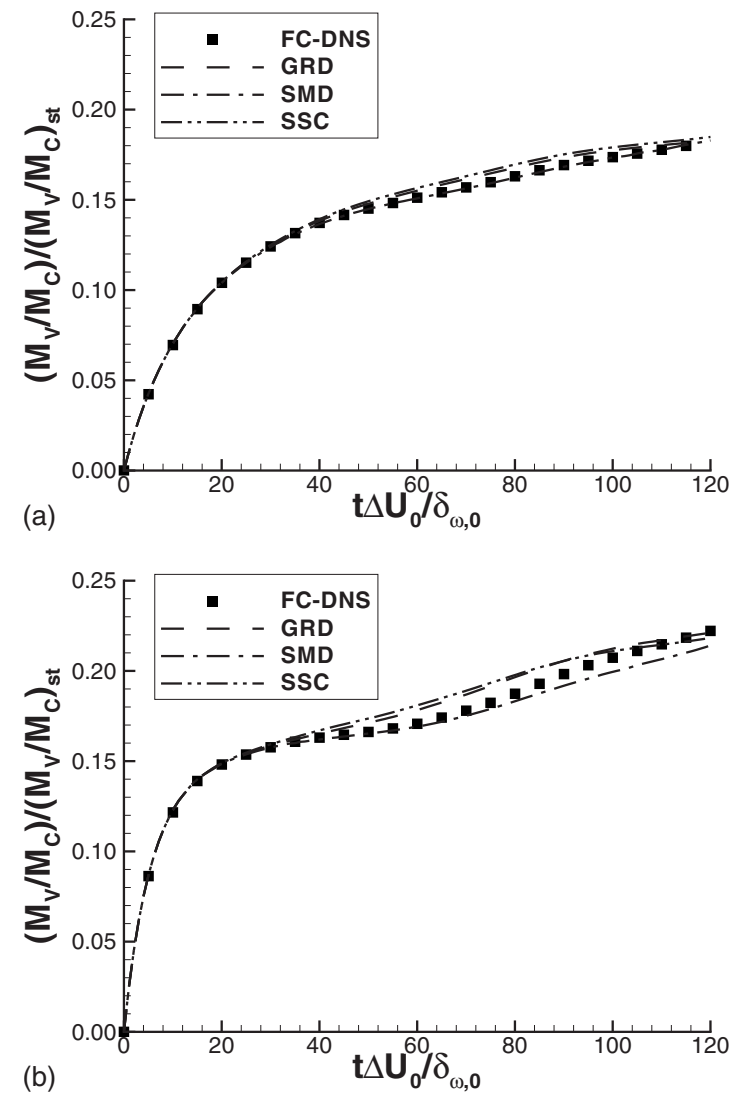

FIG. 10. Evolution of global equivalence ratio: (a) TP600a2 and (b) TP600a5.

the FC-DNS dissipation is here addressed. From the entropy equation, ${ }^{39}$ the rate of irreversible entropy production has been derived by Okong'o and Bellan ${ }^{8}$ as

$$
\begin{aligned}
& g=g_{\mathrm{III}}+g_{\mathrm{II}}+g_{\mathrm{I}, \mathrm{kine}}+g_{\mathrm{I}, \text { chpot }}+g_{\text {visc }}+g_{\text {temp }}+g_{\text {mass }}, \\
& g_{\mathrm{III}}=\frac{S_{\mathrm{III}}}{T}, \quad g_{\mathrm{II}}=-u_{i} \frac{S_{\mathrm{II}, i}}{T}, \\
& g_{\mathrm{I}, \mathrm{kine}}=\left(\frac{1}{2} u_{i} u_{i}\right) \frac{S_{I}}{T}, \quad g_{I, \text { chpot }}=-\mu_{V} \frac{S_{I}}{T},
\end{aligned}
$$

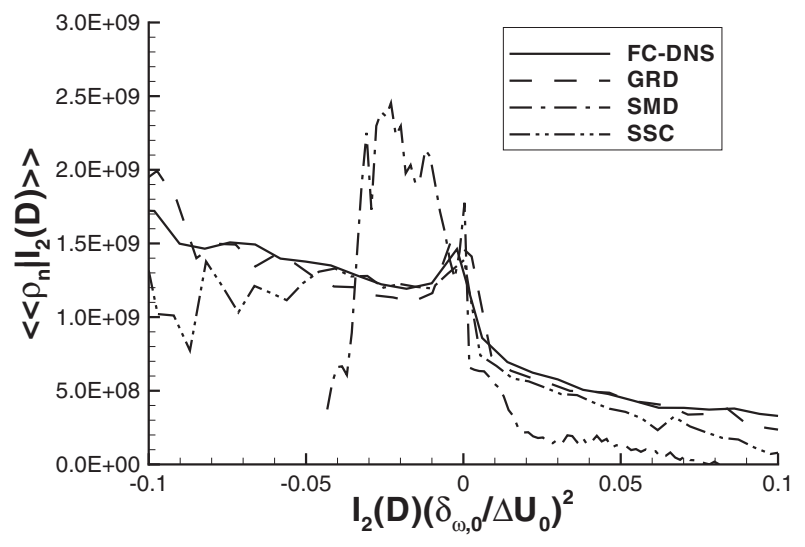

(a)

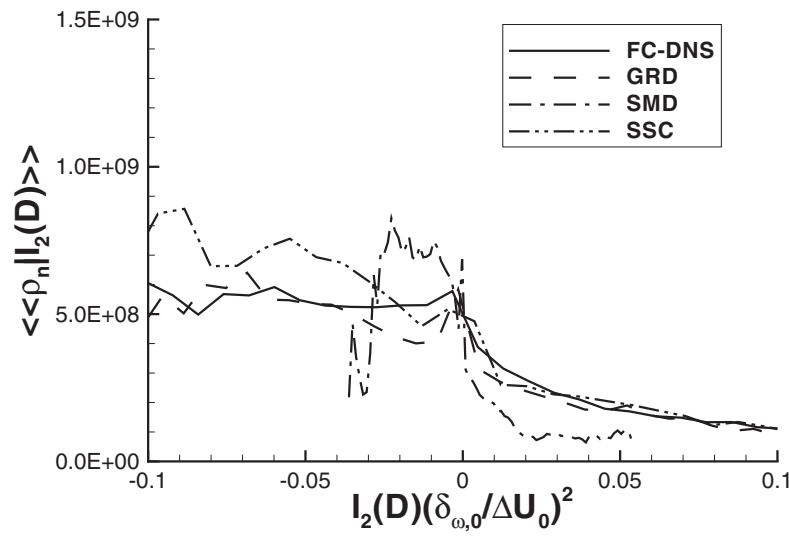

(b)

FIG. 9. Drop number density within layer $\left(\mathrm{m}^{-3}\right)$, conditionally averaged on $I_{2}(\mathbf{D})$ : (a) TP600a2 and (b) TP600a5. 
(a)
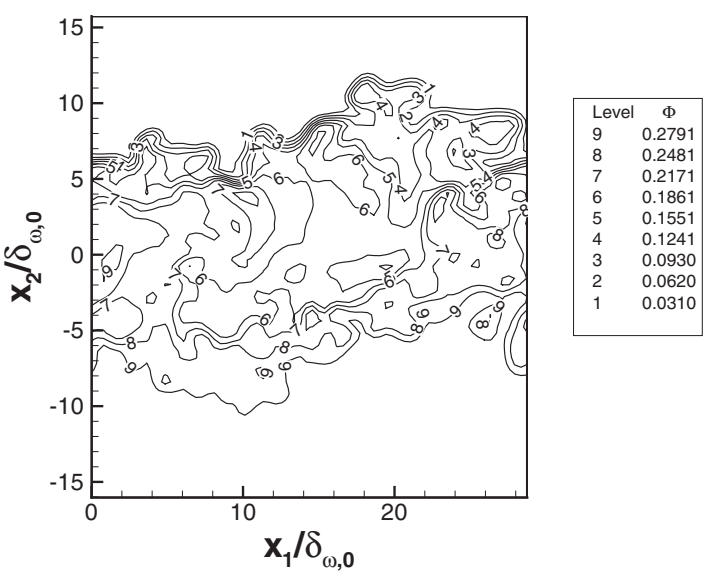

(c)
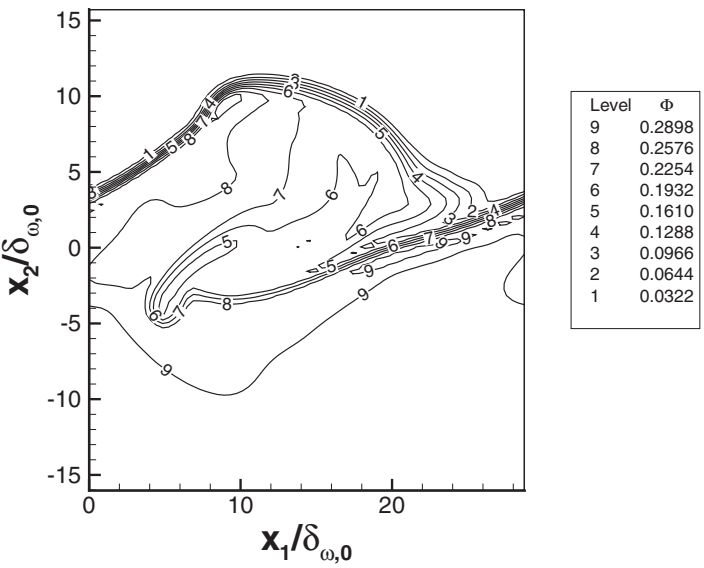
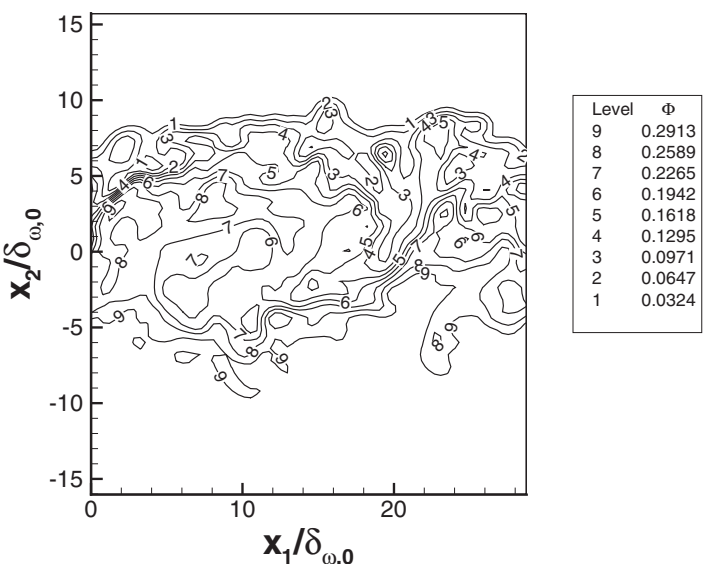

(b)

(d)

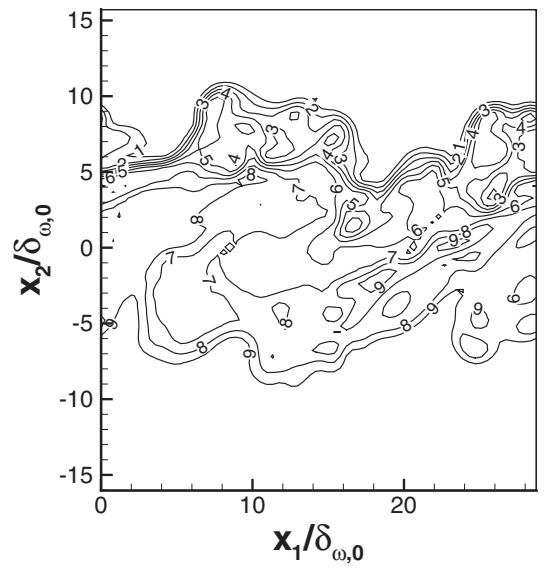

FIG. 11. Equivalence ratio, TP600a2 at $t^{*}=105$, between-the-braid plane: (a) FC-DNS, (b) GRD, (c) SMD, and (d) SSC.

$$
\begin{aligned}
& g_{\text {visc }}=\frac{2 \mu}{T}\left(S_{i j}-\frac{1}{3} S_{k k} \delta_{i j}\right)^{2}, \quad g_{\text {temp }}=\frac{\lambda}{T^{2}} \frac{\partial T}{\partial x_{j}} \frac{\partial T}{\partial x_{j}}, \\
& g_{\text {mass }}=\frac{R_{C} R_{V}}{Y_{C} Y_{V}\left(R_{V} Y_{V}+R_{C} Y_{C}\right)} \frac{j_{V j} j_{V j}}{\rho D},
\end{aligned}
$$

where $\mu_{V}$ is the chemical potential of the vapor; $\mu_{V}=h_{V}$ $-T s_{V}$. The pure vapor entropy, $s_{V}$ is here calculated as

$$
s_{V}=s_{V}^{0}+C_{p, V} \ln \left(T / T^{0}\right)-R_{V} \ln \left(p / p^{0}\right),
$$

where $s_{V}^{0}$ is the reference entropy at $\left(T^{0}, p^{0}\right)$ obtained from integration or tables. Inspection of Eq. (43) shows that the gas-phase dissipation has several origins. First, the drops are energy, momentum, and mass sources with the resulting dissipation embodied in $g_{\mathrm{III}}, g_{\mathrm{II}}, g_{\mathrm{I}, \mathrm{kine}}$ and $g_{\mathrm{I} \text {,chpot. Note that }}$ $g_{\mathrm{I}, \mathrm{kine}}$ and $g_{\mathrm{I}, \mathrm{chpot}}$ are entirely due to evaporation, with $g_{\mathrm{I}, \mathrm{kine}}$ due to the gas-phase kinetic energy of the mass evolving from the drops, whereas $g_{\mathrm{I} \text {,chpot }}$ is due to its chemical potential. Similar effects are contained in $g_{\mathrm{III}}$ and $g_{\mathrm{II}}$, but these terms additionally have nonevaporation contributions from the drag on the drops and the heating of the drops. The terms $g_{\text {visc }}, g_{\text {temp }}$ and $g_{\text {mass }}$ contain the flux-related dissipation and are positive semidefinite.

The terms in Eq. (43) are listed in Table III, which contains the domain average and rms values for TP600a2 and TP600a5 at transition in decreasing order of FC-DNS rms.
Due to extreme sensitivity of $g_{\text {mass }}$ to numerical error, the present results limit $\left|\partial\left(\ln Y_{V}\right) / \partial x_{i}\right|<2 / \Delta x_{i}$. Considering first the FC-DNS, the largest terms on average are $g_{\text {III }}$, which is positive, and $g_{\mathrm{I} \text {,chpot }}$, which is negative and also of smaller magnitude. The $g_{\text {II }}$ is also negative on average; its magnitude is larger than that of $g_{\text {visc }}$ for TP600a5 FC-DNS but smaller than $g_{\text {visc }}$ for TP600a2 FC-DNS. For both TP600a2 and TP600a5, $g_{\text {visc }}$ and $g_{\text {II }}$ are one to two orders of magnitude smaller than the largest terms. The smallest term is $g_{\text {temp }}$; of the two remaining terms ( $g_{\text {mass }}$ and $\left.g_{\mathrm{I}, \mathrm{kine}}\right), g_{\text {mass }}$ is minimally larger for TP600a 2 while $g_{\text {I,kine }}$ is larger for TP600a5. For the FC-DNS rms, TP600a2 and TP600a5 have the same ordering of terms: the largest magnitude term is $g_{\mathrm{III}}$, followed by $g_{\text {I,chpot }}$, then $g_{\text {III }}$. Next are the flux-related terms, with $g_{\text {visc }}$ being largest and $g_{\text {temp }}$ being smallest; the remaining sourcerelated term $g_{\mathrm{I}, \text { kine }}$ has an rms larger than $g_{\text {temp }}$ but smaller than $g_{\text {mass }}$.

Comparing the LES with the FC-DNS, all LESs preserve the ordering of terms of the FC-DNS rms. Generally, the ordering of terms is also preserved for the average; however, $g_{\text {II }}$ has the wrong sign for the TP600a2 SMD LES and for all TP600a5 LES, and the relative magnitudes of the $g_{\mathrm{I}, \mathrm{kine}}$ and $g_{\text {mass }}$ averages are reversed for TP600a2 SMD, TP600a5 GRD and TP600a5 SSC. Thus, $g_{\text {II }}$ and $g_{\text {mass }}$ seem most sensitive to the LES modeling errors. The proportional contribution of all terms to the total is generally the same for the 
(a)
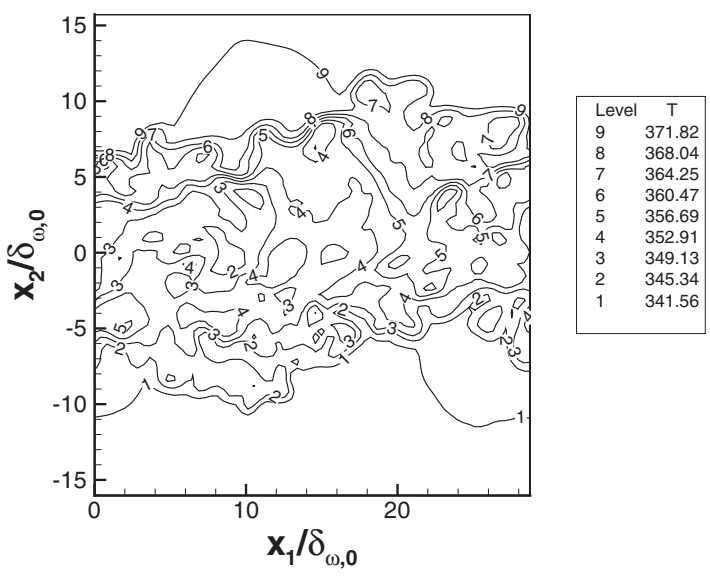

(c)
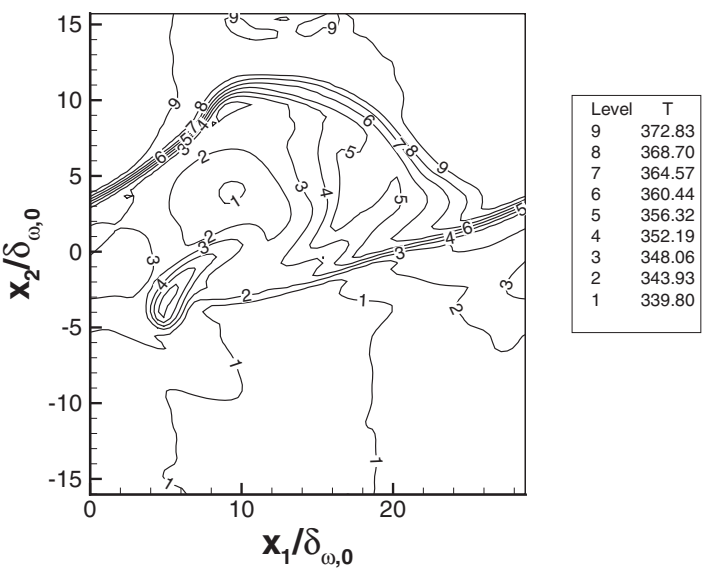
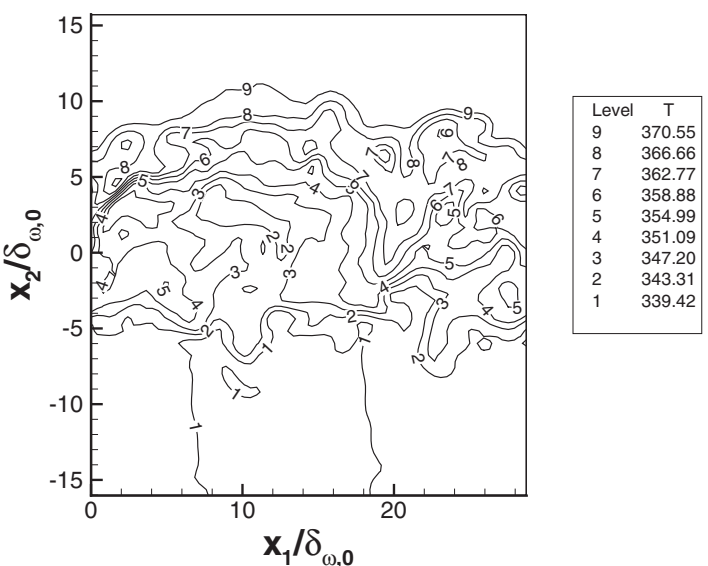

(b)

(d)

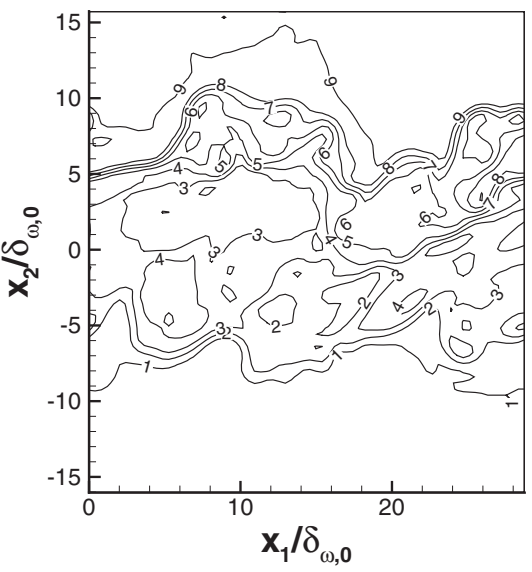

FIG. 12. Temperature (K), TP600a2 at $t^{*}=105$, between-the-braid plane: (a) FC-DNS, (b) GRD, (c) SMD, and (d) SSC.

TABLE III. Entropy production $\left(\mathrm{W} / \mathrm{m}^{3} \mathrm{~K}\right)$ of FC-DNS and LES, $t^{*}=105$.

\begin{tabular}{|c|c|c|c|c|c|c|c|c|}
\hline & \multicolumn{4}{|c|}{ Average } & \multicolumn{4}{|c|}{$\mathrm{rms}$} \\
\hline & FC-DNS & GRD & SMD & SSC & FC-DNS & GRD & SMD & SSC \\
\hline \multicolumn{9}{|c|}{ TP600a2 } \\
\hline$g_{\text {III }}$ & 17504 & 8973 & 12551 & 8787 & 33227 & 20240 & 28312 & 20424 \\
\hline$g_{\mathrm{I}, \mathrm{chpot}}$ & -10270 & -5129 & -7512 & -5105 & 20414 & 12685 & 17856 & 12859 \\
\hline$g_{\text {II }}$ & -308 & -41 & 10 & -111 & 1881 & 1715 & 1943 & 1524 \\
\hline$g_{\text {visc }}$ & 600 & 423 & 366 & 360 & 1109 & 825 & 640 & 736 \\
\hline$g_{\text {mass }}$ & 74 & 103 & 74 & 74 & 313 & 524 & 388 & 377 \\
\hline$g_{\mathrm{I}, \mathrm{kine}}$ & 73 & 30 & 85 & 23 & 191 & 147 & 274 & 141 \\
\hline$g_{\text {temp }}$ & 22 & 18 & 28 & 19 & 46 & 42 & 76 & 45 \\
\hline$g$ & 7694 & 4377 & 5603 & 4047 & 13588 & 8419 & 11409 & 8316 \\
\hline \multicolumn{9}{|c|}{ TP600a5 } \\
\hline$g_{\text {III }}$ & 33900 & 15644 & 22101 & 12464 & 58899 & 33308 & 55526 & 28605 \\
\hline$g_{\text {I,chpot }}$ & -19485 & -8269 & -12327 & -6131 & 36988 & 21454 & 35621 & 18926 \\
\hline$g_{\mathrm{II}}$ & -844 & 147 & 442 & 153 & 5553 & 4999 & 7280 & 4278 \\
\hline$g_{\text {visc }}$ & 663 & 530 & 348 & 302 & 1208 & 990 & 636 & 607 \\
\hline$g_{\text {mass }}$ & 96 & 122 & 81 & 72 & 430 & 669 & 515 & 379 \\
\hline$g_{\mathrm{I}, \mathrm{kine}}$ & 158 & 45 & 107 & 13 & 393 & 261 & 464 & 228 \\
\hline$g_{\text {temp }}$ & 26 & 20 & 24 & 17 & 58 & 50 & 84 & 44 \\
\hline$g$ & 14514 & 8240 & 10778 & 6889 & 22849 & 13843 & 23024 & 11353 \\
\hline
\end{tabular}


(a)
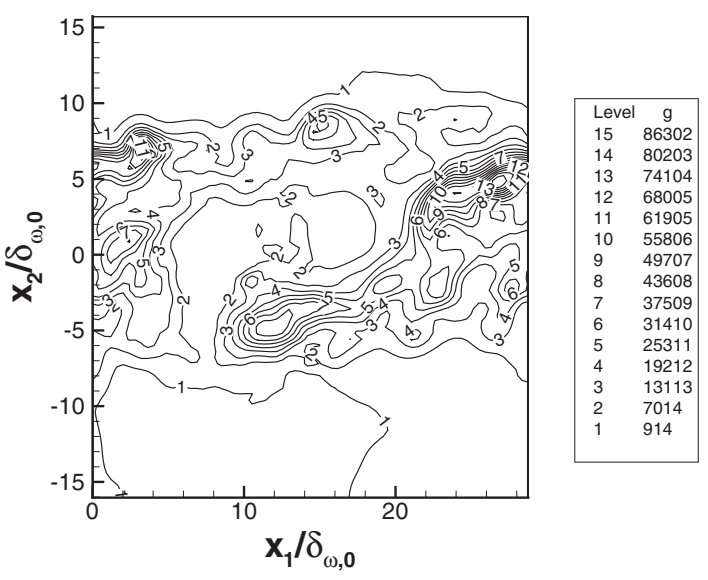

(c)
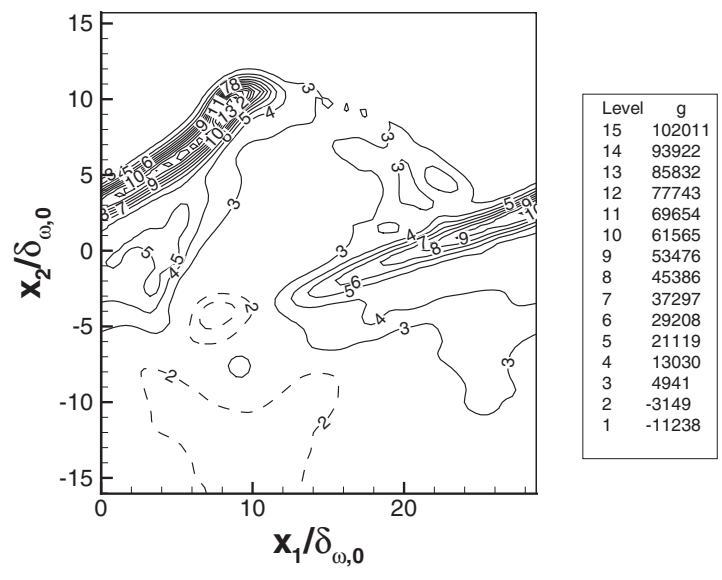
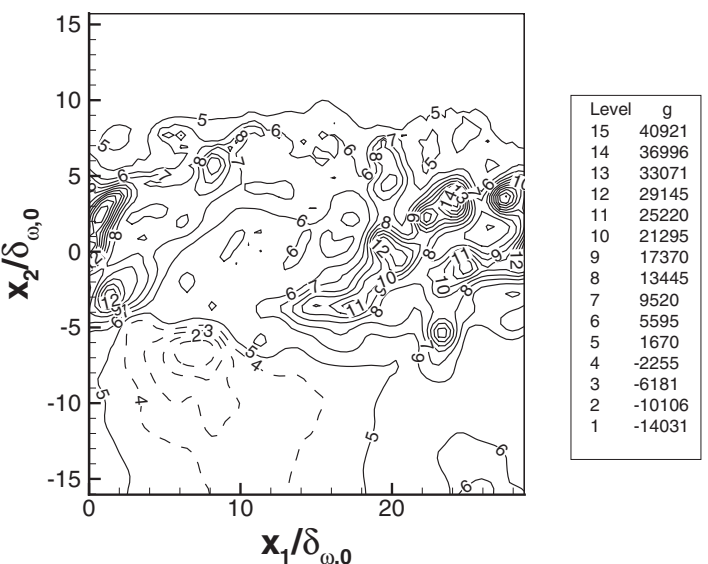

(b)

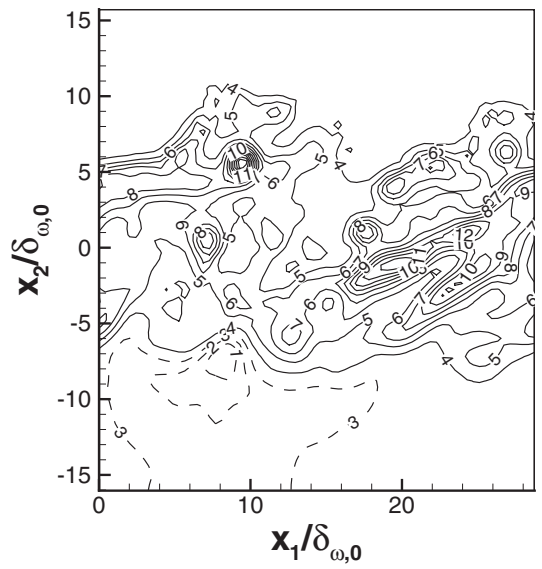

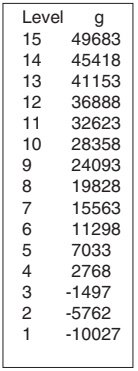

(d)

FIG. 13. Dissipation (W/ $/ \mathrm{m}^{3} \mathrm{~K}$ ), TP600a2 at $t^{*}=105$, between-the-braid plane: (a) FC-DNS, (b) GRD, (c) SMD, and (d) SSC. Dashed lines denote negative values.

LES and the FC-DNS, however, the magnitude of the LES terms varies considerably from the FC-DNS. In particular, the average $g$ for the LES is lower than the FC-DNS, being about 50\%-60\% for GRD and SSC and about $75 \%$ for SMD; the largest magnitude terms, $g_{\text {IIII }}$ and $g_{\mathrm{I} \text {,chpot }}$, are typically even lower, $30 \%-50 \%$ for GRD and SSC and $60 \%-75 \%$ for the SMD. The most obvious reason for the reduced source values in LES compared to the FC DNS is the disparity in the representation of the drop field due to the different numbers of drops (see Table II) and smaller size drops (see Fig. 7) which combine to reduce the source terms.

Since the dissipation is dominated by source-term contributions and, as already discussed in conjunction with Fig. 10 , the global amount of evaporation is well predicted by all models, it turns out that the global dissipation results are generally insensitive to the choice of the SGS model. This finding explains previous success of other researchers with the SMD model, ${ }^{16,20}$ as it better predicts the global amount of dissipation compared to the GRD and SSC models. However, the location of the dissipation predicted by the SMD LES is not correct, as seen on the contour plots in Fig. 13. As previously observed for other quantities (Figs. 3, 11, and 12), the SMD model is devoid of small-scale structure, while the GRD and SSC have similar small-scale structure to the FCDNS. Because $g$ is dominated by source terms, which result from the drop locations (Fig. 6), the SGS model does affect the local dissipation distribution. The range of $g$ values is larger for the SMD model compared to the FC-DNS, while the range is smaller for the GRD and SSC versus the FCDNS. The implication is that the SMD model produces less spatial extent but more intensity of high dissipation regions coinciding with the overly high drop accumulation, leading in the present cases to global average dissipation that is comparable to that of the other LES models.

\section{CONCLUSIONS}

Results from LES of a temporal mixing layer with evaporating drops have been compared with those from FC DNS in order to assess the ability of the LES to replicate detailed aspects of the DNS at the LES scale. The LESs were conducted with models for the filtered source and the SGSflux terms which result from filtering of the DNS equations. Three different SGS-flux models were evaluated: dynamic gradient (GRD), dynamic Smagorinsky (SMD), and constant-coefficient scale similarity (SSC). The dynamic models used model coefficients which were computed during LES from the LES solution, while the SSC model used a constant-coefficient value calibrated on the DNS database. The LES used a reduced computational drop field, with each 
LES drop representing eight physical DNS drops, as well as a reduced flow-field resolution, with the LES grid spacing being four times that of the DNS. All LESs captured the largest-scale vortex and processes associated with it, such as the global fuel-to-vapor ratio and the range of drop sizes. A comparison of the dynamic and mixing characteristics revealed the inability of the SMD model to replicate smallscale structures in both dynamic (enstrophy) and thermodynamic (temperature, equivalence ratio) characteristics. The GRD and SSC models did capture such small-scale activity.

Although the LES did not directly incorporate SGS effects on drop evolution, the choice of the SGS model was found to affect the drop spatial distribution. The relationship between drop spatial distribution and layer dynamics was explored by conditionally averaging on the second invariant of the deformation tensor. The GRD and SSC drop spatial distributions well matched the FC-DNS, with drops most likely to be located where the invariant was close to zero. The SMD model led to a different drop spatial distribution, with the correlation of invariant and drops being qualitatively different from the FC-DNS. The impact of layer dynamics, mixing, and drop distribution on the irreversible entropy production (dissipation) was that all LESs underpredict the global amount of dissipation. Although the SMD LES best predicts the global dissipation, it proved locally inaccurate, with the spatial distribution of dissipation being better matched by the GRD and the SSC. The dissipation activity is mainly due to drop source terms.

The overall assessment is that while the SMD model does capture some global aspects, local detailed aspects are better predicted by the GRD and SSC models. Therefore the GRD and SSC models are recommended for situations, such as combustion, where the local conditions have a significant impact on the flow. The GRD model has the advantage over the SSC model of not requiring a calibrated coefficient. Future work includes incorporating direct SGS effects on the drops as proposed by the deterministic model of Okong'o and Bellan ${ }^{8}$ to potentially improve the LES results.

\section{ACKNOWLEDGMENTS}

This study was conducted at the Jet Propulsion Laboratory (JPL) of the California Institute of Technology (Caltech) under the sponsorship of the U.S. Department of Energy, with R. Danz and D. Hooker serving as contract monitors, under an agreement with the National Aeronautics and Space Administration. Computations were performed on the SGI Origin 2000 at the JPL Supercomputing Center.

\footnotetext{
${ }^{1}$ M. Boivin, O. Simonin, and K. D. Squires, "Direct numerical simulation of turbulence modulation by particles in isotropic turbulence," J. Fluid Mech. 375, 235 (1998).

${ }^{2}$ F. Mashayek and F. A. Jaberi, "Particle dispersion in forced isotropic low-Mach-number turbulence," Int. J. Heat Mass Transfer 42, 2823 (1999).

${ }^{3}$ F. Mashayek, "Direct numerical simulations of evaporating droplet dispersion in forced low-Mach-number turbulence," Int. J. Heat Mass Transfer 41, 2601 (1998).

${ }^{4}$ J. Réveillon and L. Vervisch, "Spray vaporization in nonpremixed turbulent combustion modeling: A single drop model," Combust. Flame 121, 75 (2000).

${ }^{5}$ F. Mashayek, "Droplet-turbulence interactions in low-Mach-number ho-
}

mogeneous shear two-phase flows," J. Fluid Mech. 367, 163 (1998).

${ }^{6}$ R. S. Miller and J. Bellan, "Direct numerical simulation of a confined three-dimensional gas mixing layer with one evaporating hydrocarbondroplet laden stream," J. Fluid Mech. 384, 293 (1999).

${ }^{7}$ R. S. Miller and J. Bellan, "Direct numerical simulation and subgrid analysis of a transitional droplet laden mixing layer," Phys. Fluids 12, 650 (2000).

${ }^{8}$ N. Okong'o and J. Bellan, "Consistent large eddy simulation of a temporal mixing layer laden with evaporating drops. Part 1: Direct numerical simulation, formulation and a priori analysis," J. Fluid Mech. 499, 1 (2004).

${ }^{9}$ J. Réveillon and L. Vervisch, "Analysis of weakly turbulent dilute-spray flames and spray combustion regimes," J. Fluid Mech. 537, 317 (2005).

${ }^{10}$ J. Réveillon and F.-X. Demoulin, "Effects of the preferential segregation of droplets on evaporation and turbulent mixing," J. Fluid Mech. 583, 273 (2007).

${ }^{11}$ C. Pera, J. Réveillon, L. Vervisch, and P. Domingo, "Modeling subgrid scale mixture fraction variance in LES of evaporating spray," Combust. Flame 146, 635 (2006).

${ }^{12}$ S. Menon and N. Patel, "Subgrid modeling for simulation of spray combustion in large-scale combustors," AIAA J. 44, 709 (2006).

${ }^{13} \mathrm{M}$. Valentino, X. Jiang, and H. Zhao, "A comparative RNS/LES study of transient gas jets and sprays under diesel conditions," Atomization Sprays 17, 451 (2007).

${ }^{14}$ J. Xia, K. H. Luo, and S. Kumar, "Large-eddy simulation of interactions between a reacting jet and evaporating droplets," Flow, Turbul. Combust. 80, 1386 (2008)

${ }^{15}$ J. P. Mellado, R. Friedrich, and S. Sarkar, "Modeling of filtered heat release for large eddy simulation of compressible infinitely fast reacting flows," Proc. Combust. Inst. 31, 1691 (2007).

${ }^{16}$ M. Boivin, O. Simonin, and K. D. Squires, "On the prediction of gas-solid flows with two-way coupling using large eddy simulation," Phys. Fluids 12, 2080 (2000).

${ }^{17}$ E. Deutsch and O. Simonin, "Large eddy simulation applied to the modelling of particulate transport coefficients in turbulent two-phase flows," Eighth Symposium on Turbulent Shear Flows (Technical University of Munich, Munich, 1991), p. 10.

${ }^{18}$ O. Simonin, E. Deutsch, and M. Boivin, "Large eddy simulation and second-moment closure of particle fluctuating motion in two-phase turbulent shear flows," Turbulent Shear Flows 9, 85 (Springer-Verlag, Berlin, 1995).

${ }^{19}$ W. Uijttewaal and R. Oliemans, "Particle dispersion and deposition in direct numerical and large eddy simulations of vertical pipe flows," Phys. Fluids 8, 2590 (1996).

${ }^{20} \mathrm{Q}$. Wang and K. D. Squires, "Large eddy simulations of particle-laden turbulent channel flow," Phys. Fluids 8, 1207 (1996).

${ }^{21}$ Y. Yamamoto, M. Potthoff, T. Tanaka, T. Kajishima, and Y. Tsuji, "Largeeddy simulation of turbulent gas-particle flow in a vertical channel: Effect of considering inter-particle collisions," J. Fluid Mech. 442, 303 (2001).

${ }^{22}$ A. Leboissetier, N. Okong'o, and J. Bellan, "Consistent large eddy simulation of a temporal mixing layer laden with evaporating drops. Part 2: A posteriori analysis," J. Fluid Mech. 523, 37 (2005).

${ }^{23}$ G. Erlebacher, M. Y. Hussaini, C. G. Speziale, and T. A. Zang, "Toward the large-eddy simulation of compressible turbulent flows," J. Fluid Mech. 238, 155 (1992)

${ }^{24} \mathrm{C}$. Fureby, "On subgrid scale modeling in large eddy simulations of compressible fluid flow," Phys. Fluids 8, 1301 (1996).

${ }^{25}$ M. Martin, U. Piomelli, and G. Candler, "Subgrid-scale models for compressible large-eddy simulations," Theor. Comput. Fluid Dyn. 13, 361 (2000).

${ }^{26} \mathrm{~N}$. Okong'o and J. Bellan, "A priori subgrid analysis of temporal mixing layers with evaporating droplets," Phys. Fluids 12, 1573 (2000).

${ }^{27}$ C. Crowe, J. Chung, and T. Troutt, "Particle mixing in free shear flows," Prog. Energy Combust. Sci. 14, 171 (1998).

${ }^{28}$ T. J. Poinsot and S. K. Lele, "Boundary conditions for direct simulations of compressible viscous flows," J. Comput. Phys. 101, 104 (1992).

${ }^{29}$ R. D. Moser and M. M. Rogers, "Mixing transition and the cascade to small scales in a plane mixing layer," Phys. Fluids A 3, 1128 (1991).

${ }^{30}$ C. A. Kennedy and M. H. Carpenter, "Several new numerical methods for compressible shear layer simulations," Appl. Numer. Math. 14, 397 (1994).

${ }^{31}$ J. Smagorinksy, "Some historical remarks on the use of nonlinear viscosities," in Large Eddy Simulation of Complex Engineering and Geophysical Flows, edited by B. Galperin and S. A. Orszag (Cambridge University Press, Cambridge, 1993) Chap. 1, pp. 3-36. 
${ }^{32}$ A. Yoshizawa, "Statistical theory for compressible turbulent shear flows, with the application to subgrid modeling," Phys. Fluids 29, 2152 (1986).

${ }^{33}$ R. A. Clark, J. H. Ferziger, and W. C. Reynolds, "Evaluation of subgridscale models using an accurately simulated turbulent flow," J. Fluid Mech. 91, 1 (1979).

${ }^{34}$ J. Bardina, J. H. Ferziger, and W. C. Reynolds, "Improved subgrid scale models for large eddy simulation," AIAA Paper No. 80-1357 (1980).

${ }^{35}$ M. Germano, U. Piomelli, P. Moin, and W. Cabot, "A dynamic subgridscale eddy viscosity model," Phys. Fluids A 3, 1760 (1991).
${ }^{36}$ B. Vreman, B. Geurts, and H. Kuerten, "Large-eddy simulation of the turbulent mixing layer," J. Fluid Mech. 339, 357 (1997).

${ }^{37}$ D. K. Lilly, "A proposed modification of the Germano subgrid-scale closure method," Phys. Fluids A 4, 633 (1992).

${ }^{38}$ K. D. Squires and J. K. Eaton, "Preferential concentration of particles by turbulence," Phys. Fluids A 3, 1169 (1991).

${ }^{39}$ J. O. Hirshfelder, C. F. Curtis, and R. B. Bird, Molecular Theory of Gases and Liquids (Wiley, New York, 1954). 